\title{
基于控制理论的透平叶栅气动反设计优化
}

\author{
丰镇平 ${ }^{*}$, 厉海涛，宋立明，李颖晨 \\ 西安交通大学能源与动力工程学院叶轮机械研究所, 西安 710049 \\ *E-mail: zpfeng@mail.xjtu.edu.cn
}

收稿日期: 2012-02-24; 接受日期: 2012-12-03

国家自然科学基金(批准号: 50776065)资助项目

\begin{abstract}
摘要本文应用控制理论, 采用提出的基于网格节点位置坐标直接变分法, 研究建立了一 般性优化问题的伴随系统，研究发展了基于控制理论的轴流式透平叶栅气动反设计优化方法 与系统. 该伴随系统的推导过程以尽可能的减少计算资源为宗旨, 应用分部积分公式和连续 伴随方法, 最终得到的目标泛函变分的表达式中仅仅含有网格坐标变分的边界积分项, 避免 了梯度计算过程中网格内部节点的重复生成, 相对于传统的伴随方法更进一步节省了计算资 源. 伴随系统的数值求解采用 $\mathrm{ROE}$ 格式近似黎曼通量和显式五步龙格-库塔时间推进法, 并 使用多重网格技术和当地时间步长加速收玫. 为验证本文伴随系统的稳定性、通用性、收敛 性和精确性，通过定义不同的目标函数进行了考核，研究结果表明，本文所研发的伴随系统 和反设计优化系统具有优秀的鲁棒性和高效性, 能够有效应用于轴流式透平叶栅气动反设计 优化中. 在此基础上, 结合本文所研究的气动优化理论, 建立了应用 Euler 方程和 N-S 方程的 伴随方法叶栅气动反设计优化方法与系统, 研发了轴流式叶栅的二维、三维无黏及黏性条件 下的压力反设计、等摘马赫数反设计软件, 成功进行了数值算例研究, 验证了该优化系统的 有效性和经济性.
\end{abstract}

叶栅气动优化设计通常是一个具有复杂约束环 境的多变量、非线性多目标优化问题. 当前广泛应用 的叶栅气动优化设计方法大致可分为全局优化方法 和梯度优化方法. 全局优化算法, 如进化算法 ${ }^{[1]}$ 、人 工神经元网络 ${ }^{[2]}$ 、响应面法 ${ }^{[3]}$ 等, 有着优秀的鲁棒性, 具有很强的通用性和全局寻优能力, 但是该类方法 计算量大, 需要耗费大量的计算资源和计算时间. 而 基于梯度的气动优化设计中需要计算目标函数对设 计变量的梯度, 因此如何快速高效的实现灵敏度分 析就显得尤为重要. 传统的梯度计算方法, 如有限差
分法、复变量法及线性化方法, 其计算量与设计变量 个数成正比, 且需要反复进行流场求解. 相比而言, 基于控制理论的气动优化设计方法, 其计算量与设 计变量无关, 可大大降低计算成本, 是实现叶栅敏捷 化设计的有效途径, 从而成为气动优化设计领域研 究的一个前沿方向, 深受国内外研究者的关注.

基于控制理论的气动优化设计方法, 首先由 Pironneau ${ }^{[4]}$ 于 1974 年应用到流体力学中, Jameson ${ }^{[5]}$ 于 1988 年将其应用到空气动力学外流领域并提出了 基于控制理论的气动优化设计方法. 随后，文献[6 8]

英文版发表信息： Feng Z P, Li H T, Song L M, et al. Aerodynamic inverse design optimization for turbine cascades based on control theory. Sci China Tech Sci, 2013, 56: 308-323, doi: 10.1007/s11431-012-5099-8 
在该方面进行了一系列深入的研究, 先后应用势流 方程、Euler 方程和 NS 方程对机翼、翼身组合乃至 全机进行了气动反设计、减阻优化以及超音速飞行中 的降噪研究, 目前已成为国外航空气动设计领域的 研究热点. Qiao 等人 ${ }^{[9]}$ 和唐智礼 ${ }^{[10]}$ 在翼型气动设计及 三维气动外形设计开展了相关的研究.

相对于外流而言, 叶轮机械叶栅内部流动更加 复杂, 且不同气动特性选择之间往往相互冲突, 基于 控制理论的叶栅优化设计方法在目标函数的选取及 其相应的伴随方程边界条件的推导、流量的控制、叶 片旋转的影响等方面仍有许多问题函待研究, 其理 论研究仍处于发展阶段. 国外, Yang 等人 ${ }^{[11]}$ 应用此 方法于 2003 年率先开展了叶轮机械叶柱的二维无黏 气动优化设计研究; $\mathrm{Wu}$ 等人 ${ }^{[12,13]}$ 以及 Papadimitriou 和 Giannakoglou ${ }^{[14 \sim 16]}$ 分别在 2003 年至 2008 年期间研 究开发了针对二维、三维透平叶柱叶型优化设计的连 续伴随方程求解器; Wang 和 $\mathrm{He}^{[17]}$ 于 2008 年将伴随 气动优化设计方法应用到了透平叶栅多级优化设计, 建立了旋转坐标系下流动方程的伴随系统, 采用混 合平面法处理伴随场在动静交界面的信息传递, 并 于 2009 年将伴随方法引入到了透平叶栅非定常优化 设计领域 ${ }^{[18]}$, 通过引入 CFD 理论中的 Harmonic 方法 开发了非定常伴随系统及其求解器, 研究了叶轮机 械叶栅非定常问题流动损失的灵敏度分析和叶片几 何气动阻力非定常优化问题, 这也是国外具有代表 性的研究工作.

国内方面, 作者所在的西安交通大学叶轮机械 研究所 TurboAero 研究组于 2004 年率先对基于控制 理论的叶轮机械叶栅连续伴随气动优化设计理论及 方法进行了研究, 推导了二维及三维 Euler 方程、二 维及三维 Navier-Stokes 方程的内流伴随方程及其边 界条件和灵敏度计算公式, 建立了无黏和黏性流动 条件下二维、三维叶栅的气动反设计及优化设计的数 学模型, 讨论了伴随系统的适定性及其对气动性能 的可控性; 给出了目标泛函的选取原则, 开发了具有 快速、精确灵敏度分析的三维黏性叶栅敏捷化气动反 设计及优化设计软件, 完成了透平二维、三维叶栅压 力反设计 ${ }^{[19-24]}$ 以及不同优化设计目标和复杂约束环 境下的气动优化设计 ${ }^{[25 ~ 27]}$, 取得了一定的研究进展, 为发展三维叶栅气动反设计及优化设计理论, 美定 了一定的理论基础.

本文应用控制理论中的连续伴随方法对轴流式
透平叶栅气动反设计优化的理论及其应用进行了研 究. 应用简洁、直观的基于网格节点位置坐标直接变 分法, 详细推导了一般优化问题的伴随方程及其内 流边界条件, 讨论了伴随系统求解的适定性条件; 结 合伴随变量给出了目标泛函对设计变量的梯度计算 式, 应用拟牛顿法, 发展了适用于轴流式透平叶栅的 气动反问题算法; 应用 $\mathrm{CFD}$ 技术中的 ROE 格式近似 黎曼解法以及五步龙格-库塔时间推进技术进行伴随 系统的数值求解, 讨论了伴随系统在气动反问题目 标函数梯度计算上的精度与速度; 并通过不同的数值 计算研究, 验证了本文所开发的轴流式透平叶栅气动 反设计优化系统的通用性、稳定性、收敛性和精确性. 在此基础上, 详细推导了轴流式透平叶栅不同气动反 问题模型的伴随系统, 结合数值求解技术、 CFD 技术、 非均匀 $\mathrm{B}$ 样条技术、网格自动生成技术以及网格扰 动技术, 应用 $\mathrm{C}++$ 语言开发了基于控制理论的轴流 式透平叶栅气动反设计优化软件, 并将其成功应用 于不同的叶栅气动反设计问题的数值算例中, 计算 结果表明该软件能够有效应用于轴流式透平叶栅气 动反设计优化中.

\section{1 一般优化问题的伴随系统}

基于控制理论的气动优化算法的核心思想是将 控制理论中的伴随方法引入气动优化问题中, 将设 计问题视为最优控制问题, 实现目标函数对设计变 量梯度的快速、经济、精确的计算. 对于透平叶俩的 气动优化问题而言, 其数学描述如下:

$$
I(\alpha, \omega) \rightarrow \min _{\alpha \in \Lambda} \text {, s.t. } E(\alpha, \omega)=0,
$$

其中, $\alpha$ 是设计变量, $\Lambda$ 为设计变量的某个适当的容许 集合, $\omega$ 是状态变量, $I(\alpha, \omega)$ 是目标泛函, $E(\alpha, \omega)=0$ 表示 N-S 方程. 通过引入控制理论中的伴 随系统, 并定义伴随变量:

$\boldsymbol{\psi}=\left\{\psi_{1}, \psi_{2}, \cdots, \psi_{m}\right\}^{\mathrm{T}}$, 二维时 $m=4$; 三维时 $m=5$.

假设伴随变量满足:

$$
\boldsymbol{\psi}^{\mathrm{T}}\left(\frac{\partial E}{\partial \boldsymbol{\omega}}\right)=\frac{\partial I}{\partial \boldsymbol{\omega}},
$$

则目标函数对设计变量的梯度可最终表示为:

$$
\frac{\mathrm{d} I}{\mathrm{~d} \alpha}=-\psi^{\mathrm{T}} \frac{\partial E}{\partial \alpha}+\frac{\partial I}{\partial \alpha} \text {. }
$$

为方便伴随系统推导过程, 定义目标泛函为: 


$$
J=I-\boldsymbol{\psi}^{\mathrm{T}} \int_{\Omega}\left[\frac{\partial\left(\boldsymbol{f}_{i}-\boldsymbol{f}_{v i}\right)}{\partial x_{i}}\right] \mathrm{d} V .
$$

假设伴随变量 $\boldsymbol{\psi}$ 在叶栅通道离散网格单元内为 固定值, 对上式采用变分有:

$$
\delta J=\delta I-\int_{\Omega} \boldsymbol{\psi}^{\mathrm{T}} \delta\left[\frac{\partial\left(\boldsymbol{f}_{i}-\boldsymbol{f}_{v i}\right)}{\partial x_{i}}\right] \mathrm{d} V .
$$

针对流动变量 $\Phi$ 来讲, 其变分包含设计变量 $\alpha$ 的变分和网格节点坐标 $x_{k}$ 的变分 ${ }^{[26]}$, 应用链导法则 和一系列的推导, 可以顺利得到某流动变量空间导 数的变分表示为:

$$
\delta\left(\frac{\partial \Phi}{\partial x_{i}}\right)=\frac{\partial(\delta \Phi)}{\partial x_{i}}-\frac{\partial \Phi}{\partial x_{k}} \frac{\partial\left(\delta x_{k}\right)}{\partial x_{i}} .
$$

应当指出, (7)式贯穿于整个连续伴随系统的建 立过程, 从而成功地实现了非线性系统中流动变量 变分和网格坐标变分的分离, 避免了网格坐标变换 及其变换矩阵的求解, 同时也为有限体积法直接 应用于连续伴随系统的数值求解奠定了基础. 因 而, 伴随系统建立的关键在于如何将流动变量变 分和设计变量变分从目标泛函变分的最终表达 式中分离, 因此需要针对(6)式的右端项逐一进行 分析.

\section{$1.1 \quad \mathrm{~N}-\mathrm{S}$ 方程变分项}

(6)式中右端第二项包含有流动变量空间导数的 变分, 需要应用(7)式进行展开以便进行变量分离. 由于设计变量与叶柾叶型数据存在一一对应的关系, 而叶栅的型线又决定了叶椓通道的网格分布情况, 因此设计变量的变分可以使用网格节点位置坐标的 变分来等价代替. 将(7)式代入(6)式中右端 N-S 方程 变分项, 并应用分部积分, 有:

$$
\begin{aligned}
& -\int_{\Omega} \boldsymbol{\psi}^{\mathrm{T}} \delta\left[\frac{\partial\left(\boldsymbol{f}_{i}-\boldsymbol{f}_{\boldsymbol{v}}\right)}{\partial x_{i}}\right] \mathrm{d} V=\int_{\Omega} \frac{\partial \boldsymbol{\psi}^{\mathrm{T}}}{\partial x_{i}} \delta\left(\boldsymbol{f}_{i}-\boldsymbol{f}_{v i}\right) \mathrm{d} V \\
& -\oint_{\partial \Omega} \boldsymbol{\psi}^{\mathrm{T}} \delta\left(\boldsymbol{f}_{i}-\boldsymbol{f}_{v i}\right) n_{i} \mathrm{~d} S+\int_{\Omega} \boldsymbol{\psi}^{\mathrm{T}} \frac{\partial\left(\boldsymbol{f}_{i}-\boldsymbol{f}_{v i}\right)}{\partial x_{j}} \frac{\partial \delta x_{j}}{\partial x_{i}} \mathrm{~d} V .
\end{aligned}
$$

为推导方便, 定义 N-S 方程的 Jacobian 矩阵如 下:

$$
A_{i}=\frac{\partial \boldsymbol{f}_{i}}{\partial \boldsymbol{\omega}}, A_{v i}=\frac{\partial \boldsymbol{f}_{v i}}{\partial \boldsymbol{\omega}}, \boldsymbol{\omega}_{i}^{\prime}=\frac{\partial \boldsymbol{\omega}}{\partial x_{i}}, B=\left[B_{i j}\right]=\left[\frac{\partial \boldsymbol{f}_{v i}}{\partial\left(\boldsymbol{\omega}_{j}^{\prime}\right)}\right] .
$$

对(8)式反复应用分部积分公式, 经过一系列的
数学推导, 并结合流动方程中进出口忽略黏性影响、 固壁壁面满足无滑移条件等客观因素, 最终可得 N-S 方程的变分为:

$$
\begin{aligned}
& -\int_{\Omega} \boldsymbol{\psi}^{\mathrm{T}} \delta\left[\frac{\partial\left(\boldsymbol{f}_{i}-\boldsymbol{f}_{v i}\right)}{\partial x_{i}}\right] \mathrm{d} V \\
= & \int_{\Omega}\left[\frac{\partial \boldsymbol{\psi}^{\mathrm{T}}}{\partial x_{i}}\left(A_{i}-A_{v i}\right)+\frac{\partial}{\partial x_{j}}\left(\frac{\partial \boldsymbol{\psi}^{\mathrm{T}}}{\partial x_{i}} B_{i j}\right)\right]\left(\delta \boldsymbol{\omega}-\frac{\partial \boldsymbol{\omega}}{\partial x_{k}} \delta x_{k}\right) \mathrm{d} V \\
& +\oint_{\partial \Omega} \frac{\partial \boldsymbol{\psi}^{\mathrm{T}}}{\partial x_{i}} B_{i j} \frac{\partial \boldsymbol{\omega}}{\partial x_{k}} n_{j} \delta x_{k} \mathrm{~d} S+\oint_{\partial \Omega} \boldsymbol{\psi}^{\mathrm{T}} \frac{\partial\left(\boldsymbol{f}_{i}-\boldsymbol{f}_{\boldsymbol{v}}\right)}{\partial x_{k}} n_{i} \delta x_{k} \mathrm{~d} S \\
& -\int_{\text {wall }}\left[\psi_{i+1}\left(n_{i} \delta p-\delta \tau_{i n}\right)-\kappa \frac{\partial \psi_{m}}{\partial n} \delta T+\kappa \psi_{m} \delta\left(\frac{\partial T}{\partial n}\right)\right] \mathrm{d} S \\
& -\int_{\text {in,out }} \boldsymbol{\psi}^{\mathrm{T}} A_{i} n_{i} \delta \boldsymbol{\omega} \mathrm{d} S-\int_{\text {in,out }} \frac{\partial \boldsymbol{\psi}^{\mathrm{T}}}{\partial x_{i}} B_{i j} \delta \boldsymbol{\omega} n_{j} \mathrm{~d} S \\
& -\int_{\text {wall }}\left[\psi_{i+1} p-\boldsymbol{\psi}^{\mathrm{T}}\left(\boldsymbol{f}_{i}-\boldsymbol{f}_{\boldsymbol{v i}}\right)\right] \delta n_{i} \mathrm{~d} S .
\end{aligned}
$$

\section{2 目标函数变分项}

不失一般性, 假设叶栅气动优化问题的目标函 数表述为: $I=\int_{\Omega} M \mathrm{~d} V+\int_{\Gamma} N \mathrm{~d} S$, 即目标函数包含叶 栅通道体积分和某边界积分. 则目标函数变分可描 述为:

$$
\delta I=\int_{\Omega} \delta(M) \mathrm{d} V+\int_{\Omega} M \delta(\mathrm{d} V)+\int_{\Gamma} \delta(N) \mathrm{d} S+\int_{\Gamma} N \delta(\mathrm{d} S) .
$$

根据文献[15], 有限体积单元的变分可表示为:

$$
\delta(\mathrm{d} V)=\frac{\partial\left(\delta x_{k}\right)}{\partial x_{k}} \mathrm{~d} V .
$$

通过积分变换以及变分分离, 目标函数的变分 可改写为:

$$
\begin{aligned}
\delta I= & \int_{\Omega} \delta(M) \mathrm{d} V+\int_{\Omega} M \delta(\mathrm{d} V)+\int_{\Gamma} \delta(N) \mathrm{d} S+\int_{\Gamma} N \delta(\mathrm{d} S) \\
= & \int_{\Omega}\left[\frac{\partial M}{\partial \boldsymbol{\omega}}-\frac{\partial}{\partial x_{i}}\left(\frac{\partial M}{\partial \boldsymbol{\omega}_{i}^{\prime}}\right)\right]\left(\delta \boldsymbol{\omega}-\frac{\partial \boldsymbol{\omega}}{\partial x_{k}} \delta x_{k}\right) \mathrm{d} V \\
& -\oint_{\partial \Omega} \frac{\partial M}{\partial \boldsymbol{\omega}_{i}^{\prime}} \frac{\partial \boldsymbol{\omega}}{\partial x_{k}} n_{i} \delta x_{k} \mathrm{~d} V+\oint_{\partial \Omega} M n_{k} \delta x_{k} \mathrm{~d} V+\int_{\Gamma} \delta_{\omega}(N) \mathrm{d} S \\
& +\oint_{\partial \Omega} \frac{\partial M}{\partial \boldsymbol{\omega}_{i}^{\prime}} \delta \boldsymbol{\omega} n_{i} \mathrm{~d} S+\int_{\Gamma} \delta_{\alpha}(N) \mathrm{d} S+\int_{\Gamma} N \delta(\mathrm{d} S) .
\end{aligned}
$$

结合 N-S 方程变分表达式(10)和目标函数变分表 达式(13)，目标泛函的最终变分为: 
$\delta J=$

$\int_{\Omega}\left[\frac{\partial \boldsymbol{\psi}^{\mathrm{T}}}{\partial x_{i}}\left(A_{i}-A_{v i}\right)+\frac{\partial}{\partial x_{j}}\left(\frac{\partial \boldsymbol{\psi}^{\mathrm{T}}}{\partial x_{i}} B_{i j}\right)+\frac{\partial M}{\partial \boldsymbol{\omega}}-\frac{\partial}{\partial x_{i}}\left(\frac{\partial M}{\partial \boldsymbol{\omega}_{i}^{\prime}}\right)\right]$ $\times\left(\delta \boldsymbol{\omega}-\frac{\partial \boldsymbol{\omega}}{\partial x_{k}} \delta x_{k}\right) \mathrm{d} V$

$$
\begin{aligned}
& \text { AdjEQ } \\
& \underbrace{+\oint_{\partial \Omega} \frac{\partial M}{\partial \omega_{i}^{\prime}} \delta \omega n_{i} \mathrm{~d} S-\int_{\mathrm{in}, \text { out }} \psi^{\mathrm{T}} A_{i} n_{i} \delta \omega \mathrm{d} S+\int_{\Gamma} \delta_{\omega}(N) \mathrm{d} S} \\
& \operatorname{Adj} B C \\
& \underbrace{\left.\int_{i+1}\left[\psi_{i} \delta p-\delta \tau_{i n}\right)-\kappa \psi_{m} \delta\left(\frac{\partial T}{\partial n}\right)+\kappa \frac{\partial \psi_{m}}{\partial n} \delta T\right] \mathrm{d} S}_{\text {wall }} \\
& \text { Adj } B C \\
& \underbrace{-\int_{\text {wall }}\left[\psi_{i+1} p-\boldsymbol{\psi}^{\mathrm{T}}\left(\boldsymbol{f}_{i}-\boldsymbol{f}_{v i}\right)\right] \delta n_{i} \mathrm{~d} S-\oint_{\partial \Omega} \frac{\partial M}{\partial \boldsymbol{\omega}_{i}^{\prime}} \frac{\partial \boldsymbol{\omega}}{\partial x_{k}} n_{i} \delta x_{k} \mathrm{~d} S} \\
& \text { Grad } \\
& +\underbrace{\oint^{\mathrm{T}} \frac{\partial\left(\boldsymbol{f}_{i}-\boldsymbol{f}_{v i}\right)}{\partial x_{k}} n_{i} \delta x_{k} \mathrm{~d} S+\int_{\Gamma} \delta_{\alpha}(N) \mathrm{d} S+\int_{\Gamma} N \delta(\mathrm{d} S)}_{\partial \Omega} \\
& \text { Grad } \\
& \underbrace{+\oint_{\partial \Omega} \frac{\partial \boldsymbol{\psi}^{\mathrm{T}}}{\partial x_{i}} B_{i j} \frac{\partial \boldsymbol{\omega}}{\partial x_{k}} n_{j} \delta x_{k} \mathrm{~d} S+\oint_{\partial \Omega} M n_{k} \delta x_{k} \mathrm{~d} S}_{\text {Grad }} .
\end{aligned}
$$

最终, 通过一系列数学公式的应用和推导, 目标 泛函的变分可成功地表达成流动变量变分和网格节 点位置坐标变分之和, (14)式由 3 部分组成, 分别构 成了叶栅气动优化问题中流动方程伴随系统的 3 个 关键因素, 即: 偏微分伴随方程组 (式中标记为 $A d j E Q$ )、偏微分伴随方程组的边界条件(式中标记为 $A d j B C$ ) 和目标泛函梯度最终表达式(式中标记为 $G \mathrm{rad}$ ). 从这三个关键因素出发, 即可对轴流式叶栅 气动优化问题中的连续伴随系统进行数学描述.

\section{3 一般优化问题伴随系统描述}

根据最优控制理论, 目标泛函的梯度求解式中要 求不再出现流动变量的变分 $\delta \omega$, 从而需要假设引入 的伴随场满足所有关于流动变分的积分项系数为零.

定义(14)式中 $A d j E Q$ 项系数为零, 可得叶轮机械 叶栅气动优化问题的偏微分伴随方程组为:

$$
\frac{\partial \boldsymbol{\psi}^{\mathrm{T}}}{\partial x_{i}}\left(A_{i}-A_{v i}\right)+\frac{\partial}{\partial x_{j}}\left(\frac{\partial \boldsymbol{\psi}^{\mathrm{T}}}{\partial x_{i}} B_{i j}\right)+\frac{\partial M}{\partial \boldsymbol{\omega}}-\frac{\partial}{\partial x_{i}}\left(\frac{\partial M}{\partial \boldsymbol{\omega}_{i}^{\prime}}\right)=0 .
$$

需要特别指出的是, 在传统的连续伴随系统推 导中 ${ }^{[5,19]}$, 需要在映射的计算网格平面进行数值离散 求解, 从而能够方便地实施计算流体动力学中的有 限差分空间离散方法. 而本文所研发的连续伴随方 程的表达形式与笛卡尔坐标系下的流动方程极其相 似, 可成功地借鉴计算流体动力学中各种成熟、稳 定、高效的偏微分方程组空间离散方法, 使得连续伴 随系统的求解不仅能够方便的适用于结构化网格和非 结构化网格, 还能够提高其求解精度和收玫速度, 从 而进一步提高连续伴随方法在叶轮机械叶栅气动优化 问题中的计算效率, 为复杂流动环境下叶轮机械叶栅 的快速、精确的气动优化及反设计(如非定常流动的 设计优化、热流耦合设计优化等)奠定良好基础.

令目标泛函变分中边界积分流动变分项 (AdjEBC 项)为零, 可得伴随方程的边界条件为:

$$
\begin{aligned}
& -\int_{\text {wall }}\left[\psi_{i+1}\left(n_{i} \delta p-\delta \tau_{i n}\right)-\kappa \psi_{m} \delta\left(\frac{\partial T}{\partial n}\right)+\kappa \frac{\partial \psi_{m}}{\partial n} \delta T\right] \mathrm{d} S \\
& +\oint_{\partial \Omega} \frac{\partial M}{\partial \omega_{i}^{\prime}} \delta \omega n_{i} \mathrm{~d} S+\int_{\Gamma} \delta_{\omega}(N) \mathrm{d} S-\int_{\mathrm{in}, \text { out }} \psi^{\mathrm{T}} A_{i} n_{i} \delta \omega \mathrm{d} S=0 .
\end{aligned}
$$

从(16)式可以看出, 在固体壁面上应该满足 $\delta p$, $\delta \tau_{i n}, \delta T$ 的系数为零才能保证伴随系统的成立. 因 此在绝热壁面上给定的某个目标函数 $N$, 应该是压 力、剪切应力和温度的函数, 考虑到 $\delta T$ 的系数与 $\delta p$ 和 $\delta \tau_{i n}$ 的系数线性无关, 可将其单独假设为零, 则 $N$ 必须是压力和剪切应力的函数. 因此, 目标函数的选 取需要满足一定的原则才能保证伴随系统的适定性, 对此问题将在本文的数值应用部分给出详细的讨论, 在此不再赘述.

将伴随方程及其边界条件代入目标泛函最终变 分表达式中, 可得:

$$
\begin{aligned}
\delta J= & \oint_{\partial \Omega} \frac{\partial \boldsymbol{\psi}^{\mathrm{T}}}{\partial x_{i}} B_{i j} \frac{\partial \boldsymbol{\omega}}{\partial x_{k}} n_{j} \delta x_{k} \mathrm{~d} S+\oint_{\partial \Omega} M n_{k} \delta x_{k} \mathrm{~d} S \\
& -\oint_{\partial \Omega} \frac{\partial M}{\partial \boldsymbol{\omega}_{i}^{\prime}} \frac{\partial \boldsymbol{\omega}}{\partial x_{k}} n_{i} \delta x_{k} \mathrm{~d} S+\oint_{\partial \Omega} \boldsymbol{\psi}^{\mathrm{T}} \frac{\partial\left(\boldsymbol{f}_{i}-\boldsymbol{f}_{v i}\right)}{\partial x_{k}} n_{i} \delta x_{k} \mathrm{~d} S \\
& -\int_{\text {wall }}\left[\psi_{i+1} p-\boldsymbol{\psi}^{\mathrm{T}}\left(\boldsymbol{f}_{i}-\boldsymbol{f}_{v i}\right)\right] \delta n_{i} \mathrm{~d} S \\
& +\int_{\Gamma} \delta_{\alpha}(N) \mathrm{d} S+\int_{\Gamma} N \delta(\mathrm{d} S) .
\end{aligned}
$$

(17)式即为泛函梯度求解的最终表达式, 由此可 以看出目标泛函的梯度只是位置坐标变分的函数, 与流动变分无关, 从而实现了快速的灵敏度分析. 此 
外通过该表达式还可以看出, 即使假设的目标函数 中有区域积分项 $M$, 目标泛函的梯度求解式只包含 有边界积分的网格坐标变分, 与传统的伴随系统相

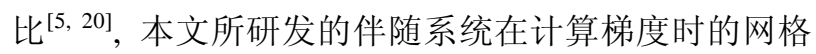
扰动中避免了反复的叶柱通道内部网格节点生成和内 部网格几何差值计算, 从而进一步节省了计算资源.

\section{2 伴随系统的数值求解技术}

由以上推导过程可知, 伴随方程的 Jacobian 矩阵 是线性化流动方程的转置. 为数值求解方便, 将其写 成时间相关形式, 最终可得直角坐标下伴随方程的 非定常形式:

$$
\frac{\partial \boldsymbol{\psi}}{\partial t}-\left(A_{i}^{\mathrm{T}}-A_{v i}^{\mathrm{T}}\right) \frac{\partial \boldsymbol{\psi}}{\partial x_{i}}-\frac{\partial}{\partial x_{j}}\left(B_{i j}^{\mathrm{T}} \frac{\partial \boldsymbol{\psi}}{\partial x_{i}}\right)-\frac{\partial M}{\partial \boldsymbol{\omega}}+\frac{\partial}{\partial x_{i}}\left(\frac{\partial M}{\partial \boldsymbol{\omega}_{i}^{\prime}}\right)=0 .
$$

本文效仿流动方程的数值解法, 采用有限容积 法求解伴随方程, 其中空间项的离散采用 ROE 格式 近似黎曼解法, 时间项的离散采用显式五步龙格-库 塔法, 并应用当地时间步长和多重网格技术进行加 速收敛.

\section{1 伴随方程的空间离散}

对于任意的有限体积单元, 伴随方程的积分形 式可写为:

$$
\begin{gathered}
\int_{\Omega} \frac{\partial \psi}{\partial t} \mathrm{~d} V-\oint_{\partial \Omega}\left(A_{i}^{\mathrm{T}}-A_{v i}^{\mathrm{T}}\right) n_{i} \psi \mathrm{d} S-\oint_{\partial \Omega} B_{i j}^{\mathrm{T}} n_{j} \frac{\partial \psi}{\partial x_{i}} \mathrm{~d} S \\
-\int_{\Omega}\left[\frac{\partial M}{\partial \boldsymbol{\omega}}-\frac{\partial}{\partial x_{i}}\left(\frac{\partial M}{\partial \boldsymbol{\omega}_{i}^{\prime}}\right)\right] \mathrm{d} V=0
\end{gathered}
$$

其中, $n_{i}$ 和 $n_{j}$ 为有限体积单元的边界法向矢量分量. 因此其半离散格式可写为:

$$
\frac{1}{\Omega} \frac{\partial \psi}{\partial t}-\sum_{\partial \Omega} \underbrace{\left[\left(A_{n}^{\mathrm{T}}-A_{v n}^{\mathrm{T}}\right) \psi \Delta S\right]}_{\text {Flux } A}-\sum_{\partial \Omega} \underbrace{\left(B_{i n}^{\mathrm{T}} \frac{\partial \psi}{\partial x_{i}} \Delta S\right)}_{\text {Flux } B}
$$

$$
-\operatorname{Source}(\boldsymbol{\psi})=0,
$$

其中, $\Omega$ 表示有限体积单元的体积, $\Delta S$ 表示有限体积单元 的边界面积, $(\cdot)_{n}=(\cdot)_{i} n_{i}$; 并定义源项为 $\operatorname{Source}(\psi)=$ $\int_{\Omega}\left[\partial M / \partial \omega-\partial\left(\partial M / \partial \omega_{i}^{\prime}\right) / \partial x_{i}\right] \mathrm{d} V$. 同时在 (20) 式中, 应用 ROE近似黎曼格式来离散伴随方程的对流项(标
记为 FluxA), 应用中心差分来离散伴随方程的扩散 项(标记为 FluxB), 即:

$$
\begin{aligned}
\text { FluxA }\left.\right|_{\partial \Omega}= & \frac{\Delta S}{2}\left\{\left.\left(A_{n}-A_{v n}\right)^{\mathrm{T}}\right|_{\mathrm{L}} \boldsymbol{\psi}_{\mathrm{L}}+\left.\left(A_{n}-A_{v n}\right)^{\mathrm{T}}\right|_{\mathrm{R}} \boldsymbol{\psi}_{\mathrm{R}}\right. \\
& \left.+\left|A_{\mathrm{Roe}}\right|^{\mathrm{T}}\left(\boldsymbol{\psi}_{\mathrm{R}}-\boldsymbol{\psi}_{\mathrm{L}}\right)\right\}, \\
\text { FluxB }\left.\right|_{\partial \Omega}= & \frac{\Delta S}{4}\left(\left.B_{i n}^{\mathrm{T}}\right|_{\mathrm{L}}+\left.B_{i n}^{\mathrm{T}}\right|_{\mathrm{R}}\right)\left(\left.\frac{\partial \boldsymbol{\psi}}{\partial x_{i}}\right|_{\mathrm{L}}+\left.\frac{\partial \boldsymbol{\psi}}{\partial x_{i}}\right|_{\mathrm{R}}\right),
\end{aligned}
$$

其中, 下标 $\mathrm{L}$ 和 $\mathrm{R}$ 分别表示边界面单元 $\partial \Omega$ 的左侧和 右侧有限体积单元; $\mid A_{\text {Roe }}$ 即为 Roe 矩阵, 其意义相同 于流动方程的 Roe 矩阵. 由此可得伴随方程的数值残 差表达式为:

$$
\begin{aligned}
\operatorname{Res}(\boldsymbol{\psi})= & \sum_{\partial \Omega} \frac{\Delta S}{2}\left\{\left.\left(A_{n}-A_{v n}\right)^{\mathrm{T}}\right|_{\mathrm{L}} \boldsymbol{\psi}_{\mathrm{L}}\right. \\
& \left.+\left.\left(A_{n}-A_{v n}\right)^{\mathrm{T}}\right|_{\mathrm{R}} \boldsymbol{\psi}_{\mathrm{R}}+\left|A_{\mathrm{Roe}}\right|^{\mathrm{T}}\left(\boldsymbol{\psi}_{\mathrm{R}}-\boldsymbol{\psi}_{\mathrm{L}}\right)\right\} \\
& +\sum_{\partial \Omega} \frac{\Delta S}{4}\left(\left.B_{i n}^{\mathrm{T}}\right|_{\mathrm{L}}+\left.B_{i n}^{\mathrm{T}}\right|_{\mathrm{R}}\right)\left(\left.\frac{\partial \boldsymbol{\psi}}{\partial x_{i}}\right|_{\mathrm{L}}+\left.\frac{\partial \psi}{\partial x_{i}}\right|_{\mathrm{R}}\right) .
\end{aligned}
$$

最终, 伴随方程的半离散格式可表示为:

$$
\frac{1}{\Omega} \frac{\partial \psi}{\partial t}=\operatorname{Res}(\boldsymbol{\psi})+\operatorname{Source}(\boldsymbol{\psi}) .
$$

\section{2 伴随方程的时间离散和多重网格技术}

本文选用显式的五步龙格-库塔法进行时间推进 求解, 其迭代形式如下:

$$
\left\{\begin{array}{l}
\psi^{0}=\psi^{n}, \\
\psi^{i}=\psi^{i-1}+\alpha_{i} \frac{\Delta t}{\Omega}\left[\operatorname{Res}\left(\psi^{i-1}\right)+\operatorname{Source}\left(\psi^{i-1}\right)\right], i=1, \cdots, 5, \\
\psi^{n+1}=\psi^{5},
\end{array}\right.
$$

其中, 龙格-库塔迭代系数为 $\alpha_{1}=1 / 4, \alpha_{2}=1 / 6, \alpha_{3}=$ $3 / 8, \alpha_{4}=1 / 2, \alpha_{5}=1$. 当地时间步长 $\left.\Delta t\right|_{\Omega}$ 定义为: $\frac{\left.\Delta t\right|_{\Omega}}{\Omega}=\frac{\mathrm{CFL}}{\Lambda_{\mathrm{c}}+C \times \Lambda_{\mathrm{v}}}, \mathrm{CFL}$ 为 Courant 数, $\Lambda_{c}$ 和 $\Lambda_{v}$ 分别 表示有限体积单元上的无黏和黏性通量特征半径之 和, 可参见文献[28]; $C$ 为常数, 本文选为 4 .

为了加快伴随方程的迭代收玫速度, 本文选取 文献[28]中的 FMG 多重网格法. 其中, 限制算子采用 粗网格单元中细网格单元的残差之和, 插值算子采 用零阶近似插值法. 


\section{3 基于控制理论的轴流式透平叶栅气动反 设计系统}

应用本文所研发的伴随系统，建立了叶栅气动 反设计最优控制模型, 结合轴流式透平叶栅的非均 匀 $\mathrm{B}$ 样条参数化、基于偏微分方程光顺的网格自动 生成、基于文本模式的商用流场求解器接口、时间推 进法求解线性伴随方程组、网格摄动和目标泛函对设 计变量梯度计算以及拟牛顿优化迭代技术, 本文研 究建立了基于控制理论的轴流式透平叶栅气动反设 计系统, 并以 $\mathrm{C}++$ 为开发工具, 研发了轴流式叶栅的 气动反设计平台.

图 1 给出了基于连续伴随方法的轴流式透平叶 栅气动优化系统流程图. 流程图的左端为用户接口, 通过输入叶栅的基本几何参数(如原始叶栅型线等) 及气动参数(如进出口条件等), 并根据具体的气动优 化问题选取设计变量及气动优化目标. 流程图的右 端给出了轴流式叶栅优化系统的具体程序模块, 这 些程序模块均通过自编程序实现并且相互独立, 具 有较强的灵活性及通用性.

为了验证本文所研发的气动反设计优化伴随系 统的稳定性, 采用同一套设计变量和网格作为考核
基准, 分别选取出口平均总压损失和出口熵增作为 目标函数进行了分析. 图 2 给出了两种不同目标函数 对设计变量的梯度的对比: 对于不同的目标函数, 由 于其物理意义相同，因而梯度的趋势完全相同，唯一 的区别在于其量级的不同, 计算结果令人满意.

图 3 给出了由伴随方法和有限差分法梯度计算 的精度对比. 相比于有限差分法, 伴随方法计算得到 的梯度误差在 $5 \%$ 以内, 梯度趋势和量级均符合很好. 验证了本文所研发的基于连续伴随方法的轴流式叶 栅气动优化伴随系统的精确性.

图 4 给出了某反问题优化的伴随场收玫史, 伴随 场的数值误差下降了 12 个数量级, 满足了反问题优 化过程中的精度要求, 验证了伴随系统的收玫性.

\section{4 叶栅反设计数值算例与验证}

\section{1 无黏条件下透平叶栅压力反设计优化}

轴流式透平叶栅的壁面压力反设计优化问题通 常给定定常流动情况下叶片表面的目标压力分布, 并通过某初始叶型采用优化手段求得与给定的目标 压力分布所对应的叶型. 同时由于三维叶栅通道内 部流动的复杂性, 无法直接给出整个叶片壁面以及

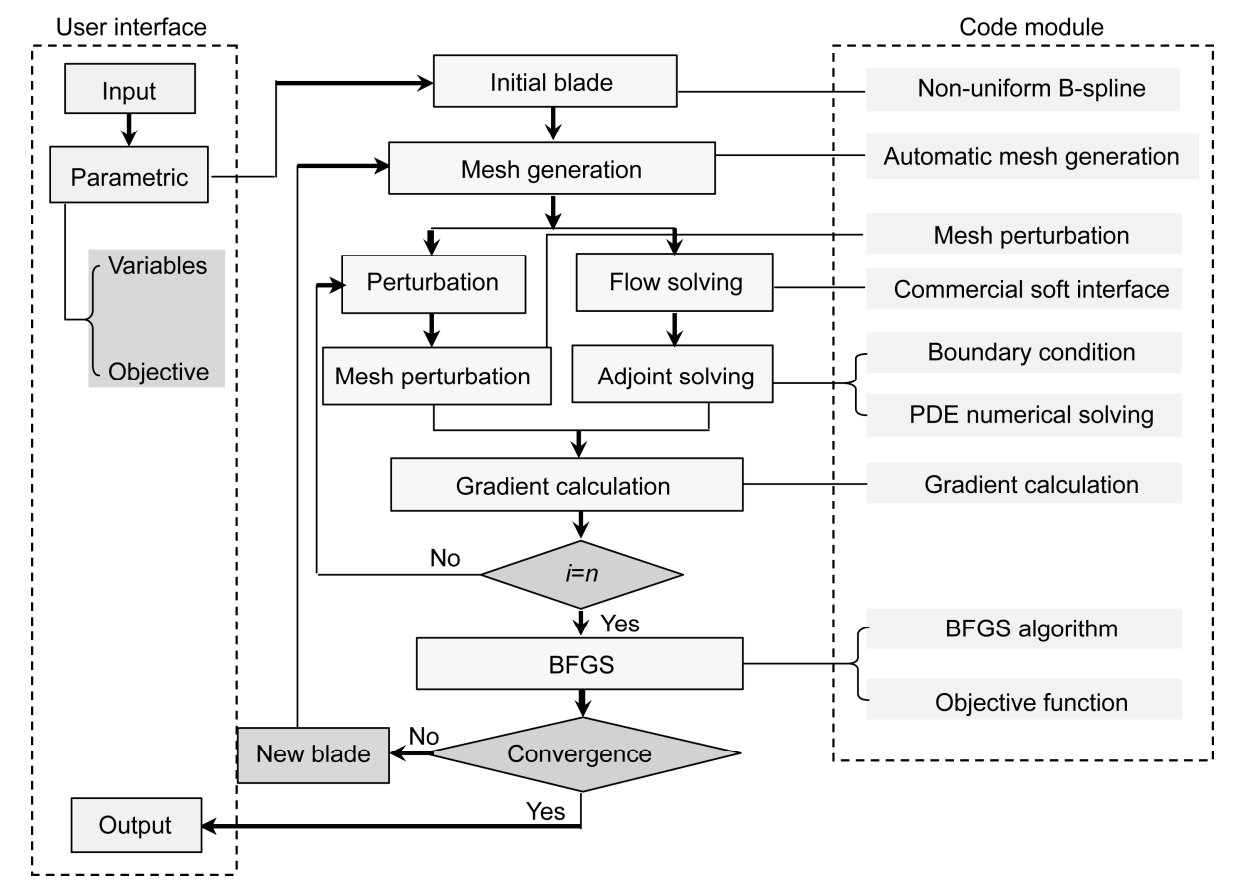

图 1 基于控制理论的轴流式透平叶栅气动反设计流程图 

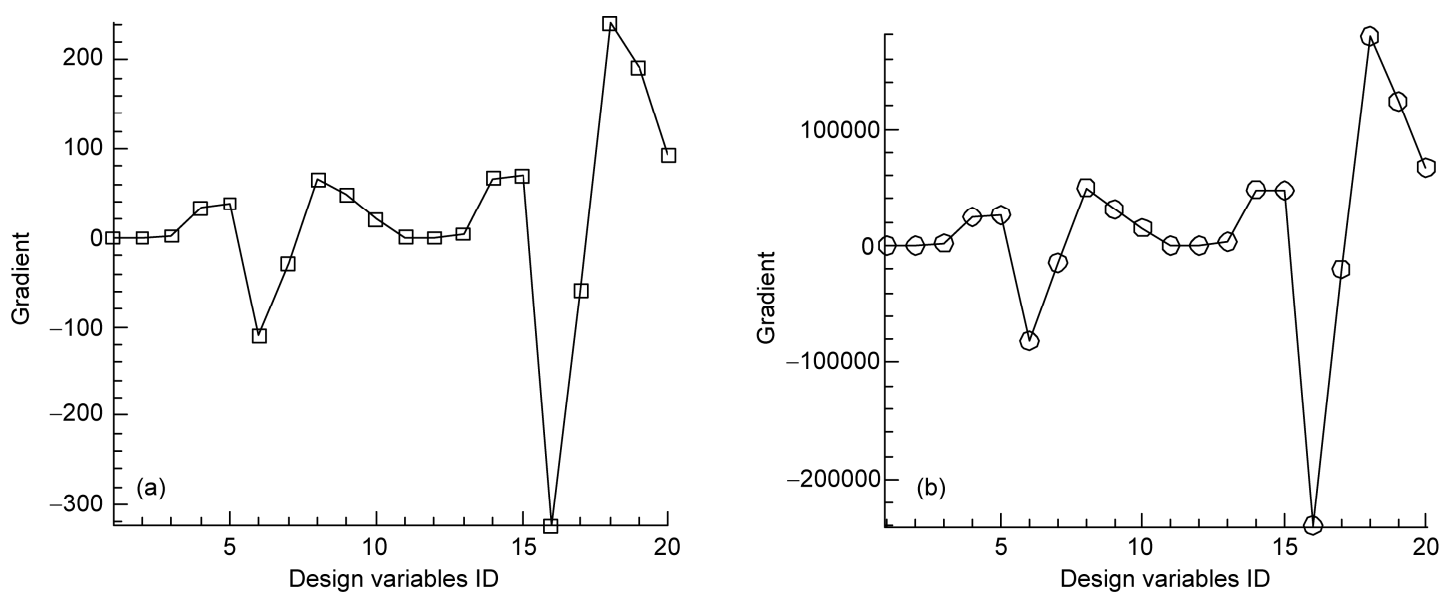

图 2 不同目标函数对设计变量梯度的对比图

(a) 出口熵增对设计变量的梯度; (b) 出口平均总压损失对设计变量的梯度
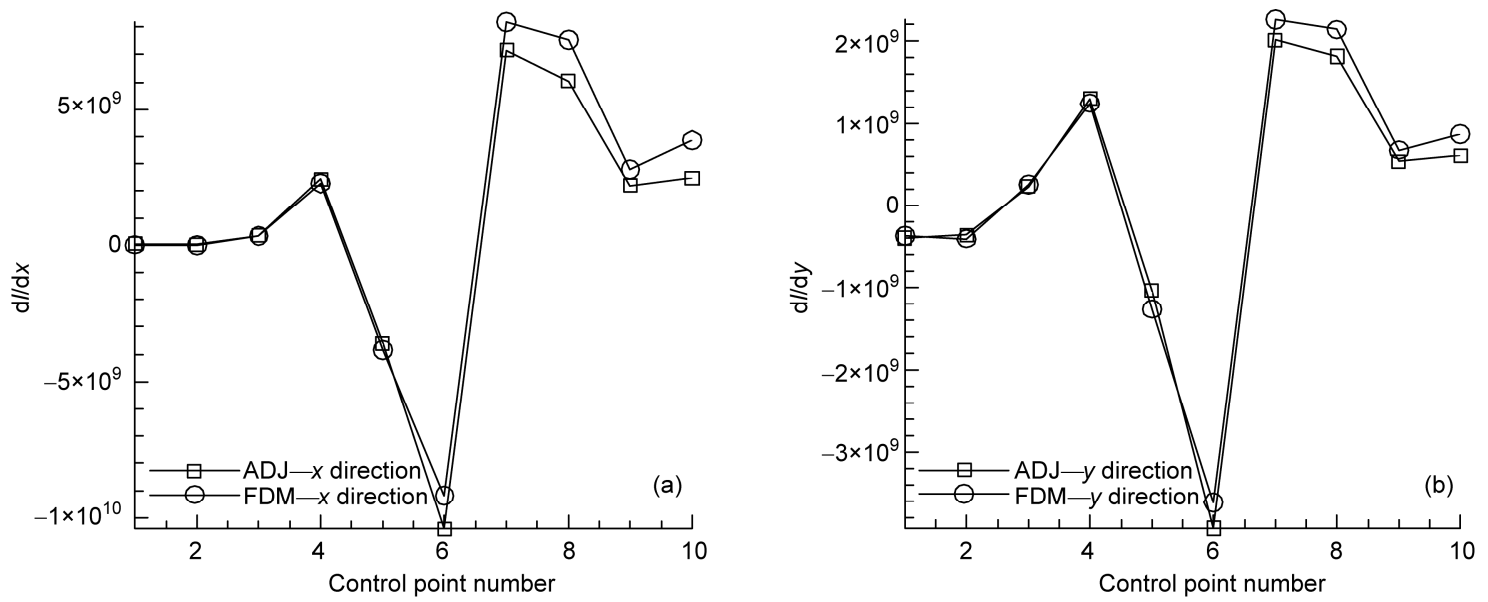

图 3 伴随方法与有限差分法计算梯度对比

(a) 设计变量 $x$ 方向目标函数梯度; (b) 设计变量 $y$ 方向目标函数梯度

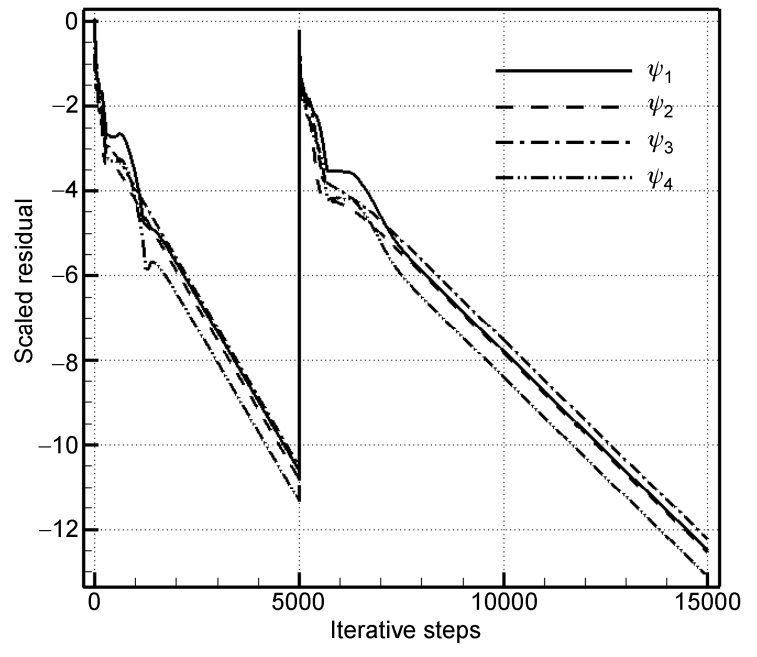

图 4 伴随场收敛史
轮缘和轮毂的压力分布, 因此在三维叶栅的压力反 设计中, 通常是通过几个关键的径向截面的压力分 布进行反设计控制, 例如给定叶片近顶部、近根部和 中径处三个典型截面上的压力分布. 对于这一问题 的数学描述, 可采用二维平面叶栅压力反设计目标 函数相同的定义方式, 但此时的压力残差最小二乘 积分是沿着给定的关键径向截面所进行的. 因此, 目 标函数的定义可选用叶片表面实际压力分布与目标 压力分布的最小二乘积分达到最小, 即:

$$
\begin{gathered}
\min _{\alpha \in \Lambda}\left\{\frac{1}{2} \int_{\text {blade }}\left(p-p_{\mathrm{d}}\right)^{2} \mathrm{~d} s, \text { s.t. } E(\alpha, \omega)=0,\right. \\
\left.\forall \vec{x}(\alpha) \in \tilde{\Omega},\left.\omega\right|_{\partial \tilde{\Omega}}=b\right\},
\end{gathered}
$$


其中, $p_{\mathrm{d}}$ 为叶片表面目标压力分布, 二维情形下 blade 表示二维叶片壁面(即吸力面和压力面); 三维情形下 blade 表示所有给定的叶片的关键径向截面(包含有吸 力面和压力面).

需要特别指出的是, 目标泛函的最终变分关于 壁面 (wall) 的积分项在二维时仅仅表示叶片壁面 (blade), 而在三维叶栅时则包含有叶片壁面(blade)、 轮教(hub)和轮缘(shroud). 本节以三维 Euler 方程为 例进行压力反设计伴随系统的推导, 二维条件下伴 随系统的建立与三维类似, 仅需忽略叶高方向的维 度即可.

将目标函数(26)式引入本文建立的伴随系统中, 最终得到的叶棚无黏条件下压力反设计的伴随系统 描述如下.

叶栅压力反设计的伴随方程为:

$$
A_{1}^{\mathrm{T}} \frac{\partial \boldsymbol{\psi}}{\partial x}+A_{2}^{\mathrm{T}} \frac{\partial \boldsymbol{\psi}}{\partial y}+A_{3}^{\mathrm{T}} \frac{\partial \boldsymbol{\psi}}{\partial z}=0,
$$

其中伴随方程 Jacobian 矩阵的具体形式可参阅文献 [23], 在此不再赘述.

伴随系统的边界条件为:

$$
\begin{aligned}
& -\int_{\text {in,out }} \boldsymbol{\psi}^{\mathrm{T}}\left(A_{1} n_{x}+A_{2} n_{y}+A_{3} n_{z}\right) \delta \boldsymbol{\omega} \mathrm{d} S \\
& +\int_{\text {blade }}\left(p-p_{\mathrm{d}}\right) \delta p \mathrm{~d} S \\
& -\int_{\text {blade,hub,shroud }}\left(\psi_{2} n_{x}+\psi_{3} n_{y}+\psi_{4} n_{z}\right) \delta p \mathrm{~d} S=0 .
\end{aligned}
$$

对于某设计变量, 压力反设计目标函数相对于 其梯度的最终表达式可写成:

$$
\begin{aligned}
\frac{\mathrm{d} J}{\mathrm{~d} \alpha}= & -\int_{\text {blade,hub,shroud }}\left(\psi_{i+1} p-\boldsymbol{\psi}^{\mathrm{T}} \boldsymbol{f}_{i}\right) \frac{\delta n_{i}}{\delta \alpha} \mathrm{d} S \\
& +\frac{1}{2} \int_{\text {blade }}\left(p-p_{\mathrm{d}}\right)^{2} \frac{\delta(\mathrm{d} S)}{\delta \alpha} \\
& +\oint_{\partial \Omega} \boldsymbol{\psi}^{\mathrm{T}} \frac{\partial \boldsymbol{f}_{i}}{\partial x_{k}} n_{i} \frac{\delta x_{k}}{\delta \alpha} \mathrm{d} S .
\end{aligned}
$$

\section{1) 二维平面叶栅压力反设计算例}

以某后加载叶栅为例, 在给定基本叶型几何参 数的情况下进行给定表面压力分布的气动反设计. 表 1 给出了叶栅的部分几何与气动参数. 图 5 给出了 初始叶型及样条控制点, 设计过程中前缘及尾缘控 制点固定.

在叶栅吸力面、压力面分别选择 4 个和 3 个可调 控制点的 $x$ 和 $y$ 坐标作为控制变量, 共计 14 个设计 变量. 计算区域采用 $\mathrm{H}$ 网格剖分, 网格数为: $85 \times 25$.
Euler 求解器采用 Fulent 6.0, 整个设计过程总共计算 了 21 遍流场, 经过 15 步设计迭代后, 无量刚目标函 数值由 0.04153 降到 0.00005 , 下降了 3 个数量级. 图 6 给出了最后迭代步的敏感性梯度, 其各个分量都接 近于零, 表明该最优设计过程已收玫至局部最优.

图 7 给出了迭代收玫过程, 图 8 给出了优化前 后叶型对比图. 图 9 给出了压力分布对比图, 可以

\section{表 1 二维叶栅的部分几何与气动参数}

\begin{tabular}{lc}
\hline \multicolumn{1}{c}{ Item } & Value \\
\hline Inlet total pressure $(\mathrm{Pa})$ & 111807.0 \\
Inlet total temperature $(\mathrm{K})$ & 311.05 \\
Outlet static pressure $(\mathrm{Pa})$ & 97700.0 \\
Pitch $(\mathrm{mm})$ & 24.92 \\
Inlet flow angle $\left({ }^{\circ}\right)$ & 90.0 \\
\hline
\end{tabular}

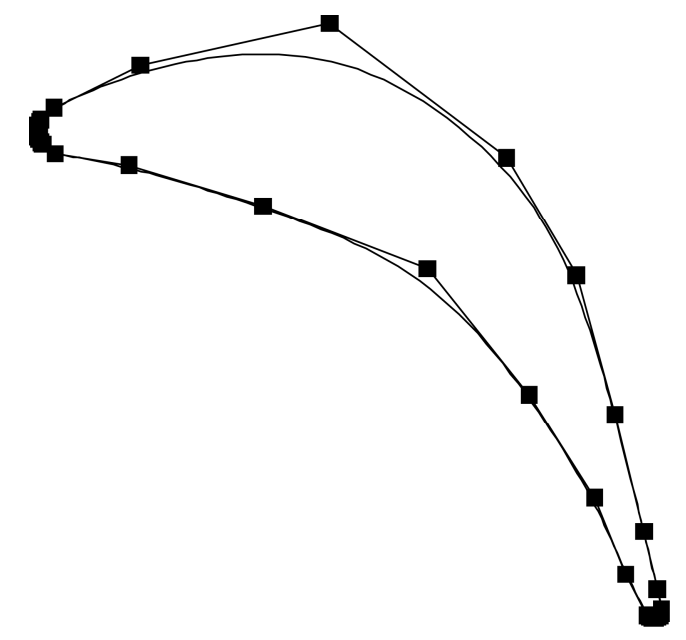

图 5 初始叶型及控制点

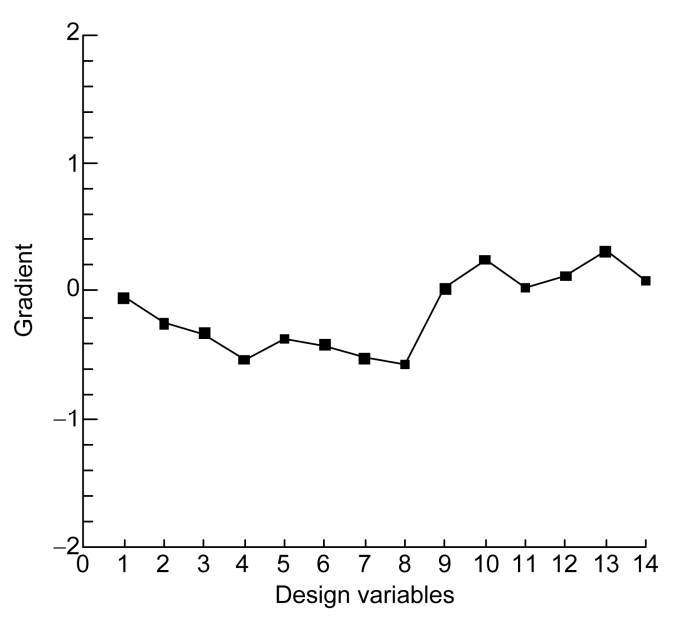

图 6 最后迭代步的敏感性梯度 


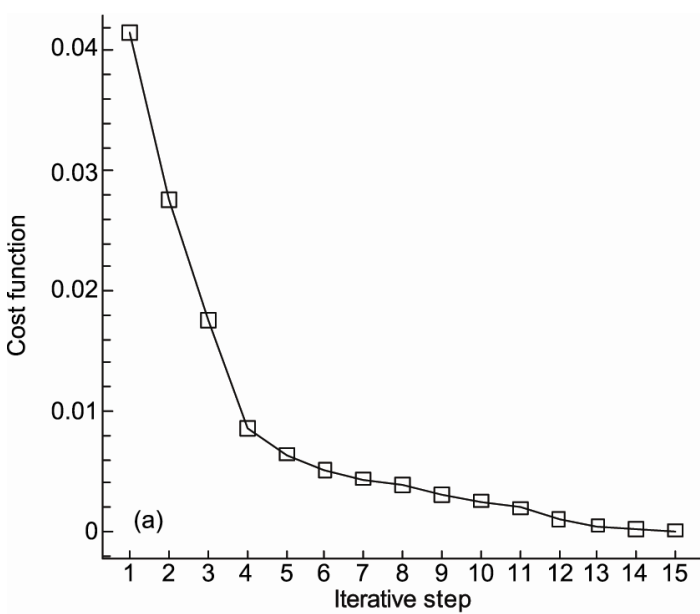

(b)

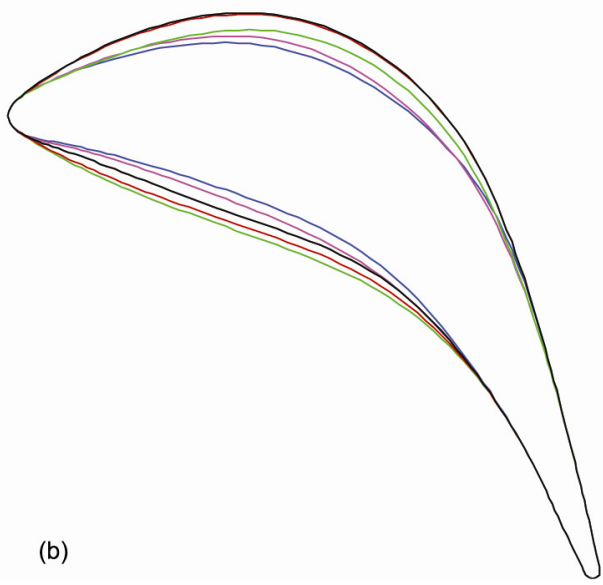

图 7 设计迭代过程

(a) 迭代收敛史; (b) 叶型变化过程

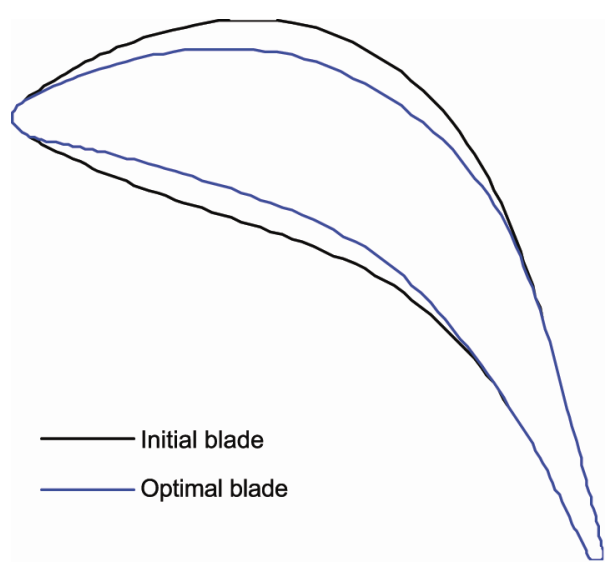

图 8 叶型设计结果

看出最优叶型对应的压力分布与目标压力分布吻合 很好.

\section{2) 三维环形叶栅压力反设计算例}

本算例通过给定叶栅近根部、中径处、近顶部的 三个截面的目标压力分布，反设计叶片三维形状. 叶 片的基本几何参数及气动参数见表 2 所示. 应用非均 匀 B 样条曲面对参考叶片进行拟合, 选取根部、中径 处及顶部截面作为设计截面，并选取其吸力面、压力 面各 10 个控制点(见图 10 的 11 号至 30 号控制点)作 为三维控制变量, 共计 60 个控制变量. 设计过程中 保证叶片尾缘半径不变. 流场数值计算采用 ANSYS CFX 11.0 求解器; 计算区域的 H-O-H 型多块结构化 网格采用自编程序自动生成.

图 11 给出了目标函数迭代收敛史, 其中目标函 数值从 54179.9 变化到 74.96 , 下降了三个数量级, 最 优叶型相比于初始叶型的压力残差明显减小. 整个 设计过程共经过 14 步反设计优化迭代，随着迭代步 的增加, 目标泛函不再有明显的下降, 迭代收玫至局 部最优点. 此外, 在整个反设计优化过程中总共计算 流场 30 次、伴随场 14 次，设计所花费的时间较少，充 分体现出基于连续伴随方法气动优化的优越性，所 设计的最终叶型及压力分布是令人满意的. 该算例

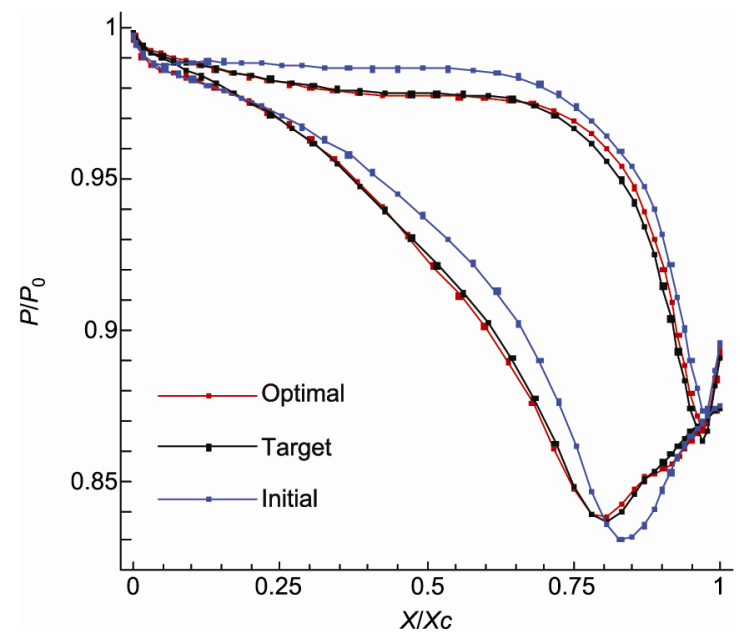

图 9 压力分布的比较

表 2 三维叶栅的部分几何与气动参数

\begin{tabular}{lc}
\hline \multicolumn{1}{c}{ Item } & Value \\
\hline Inlet total pressure $(\mathrm{Pa})$ & 344740.0 \\
Inlet total temperature $(\mathrm{K})$ & 709.0 \\
Number of blades & 45 \\
Middle diameter $(\mathrm{m})$ & 0.70 \\
Blade height $(\mathrm{m})$ & 0.04 \\
Outlet static pressure $(\mathrm{Pa})$ & 206431.0 \\
Inlet flow angle $\left({ }^{\circ}\right)$ & 90.0 \\
\hline
\end{tabular}




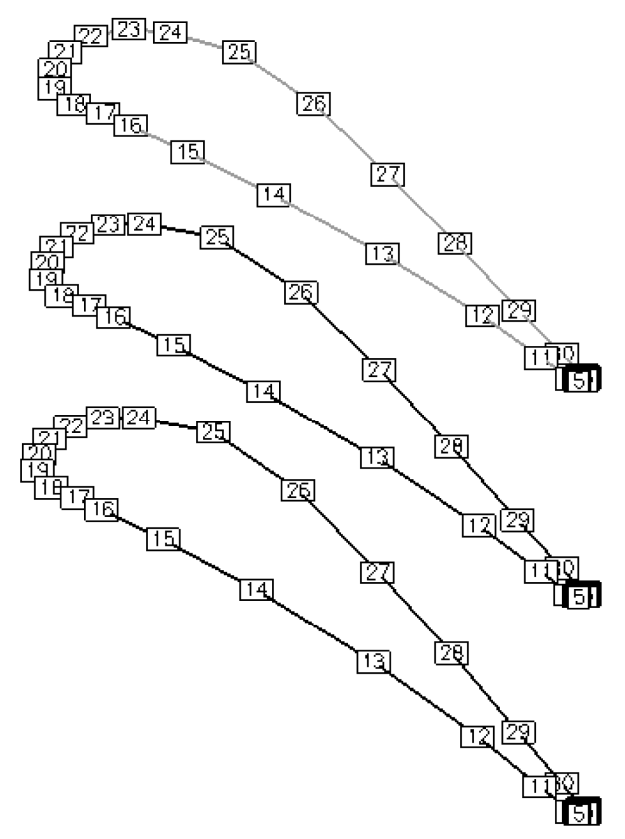

图 10 原始叶片及其控制点

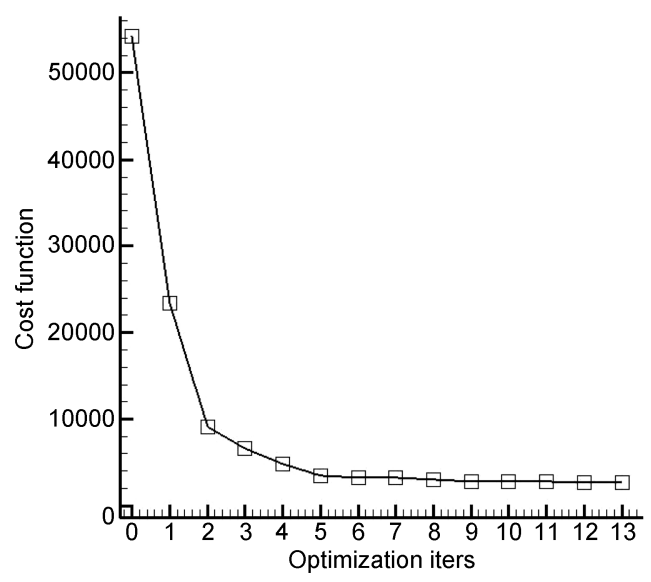

图 11 目标函数收玫图

的研究结果表明本文所研发的无黏条件下三维环形 叶栅压力反设计程序可高效实现三维叶栅的气动反 设计.

由于该算例属于跨音速流动叶栅, 目标函数相 对于设计变量的空间分布极其复杂, 其梯度值亦具 有较高的灵敏性, 从而使得反设计过程中叶片表面 微小的改动也将引起压力分布明显的变化, 因此反 设计优化前后的叶型变化非常小, 而从图 12 给出的 压力分布与目标值的比较中可看出在根部、中径处及 顶部截面，除吸力面出口附近的压力稍有波动之外,
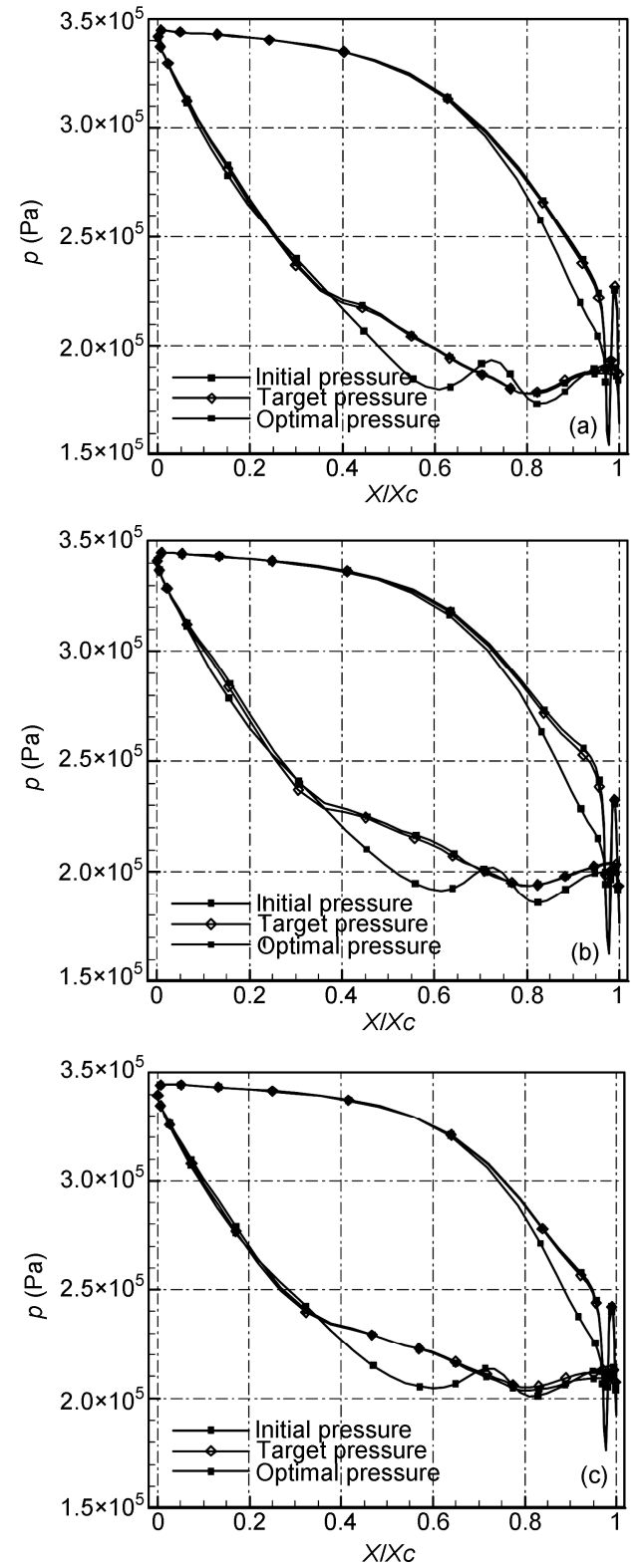

图 12 叶片三个典型截面的压力分布对照图 (a) 近根部压力分布; (b) 中径处压力分布; (c) 近顶部压力分布

压力分布基本吻合. 图 13 给出了最终迭代步与初始 迭代步的敏感性梯度分量对比图, 三个关键截面的 梯度均接近于零, 说明优化进程已很好收玫.

\section{2 黏性条件下透平叶栅压力反设计优化}

与无黏条件下的压力反设计类似, 同样定义目 标函数为: 

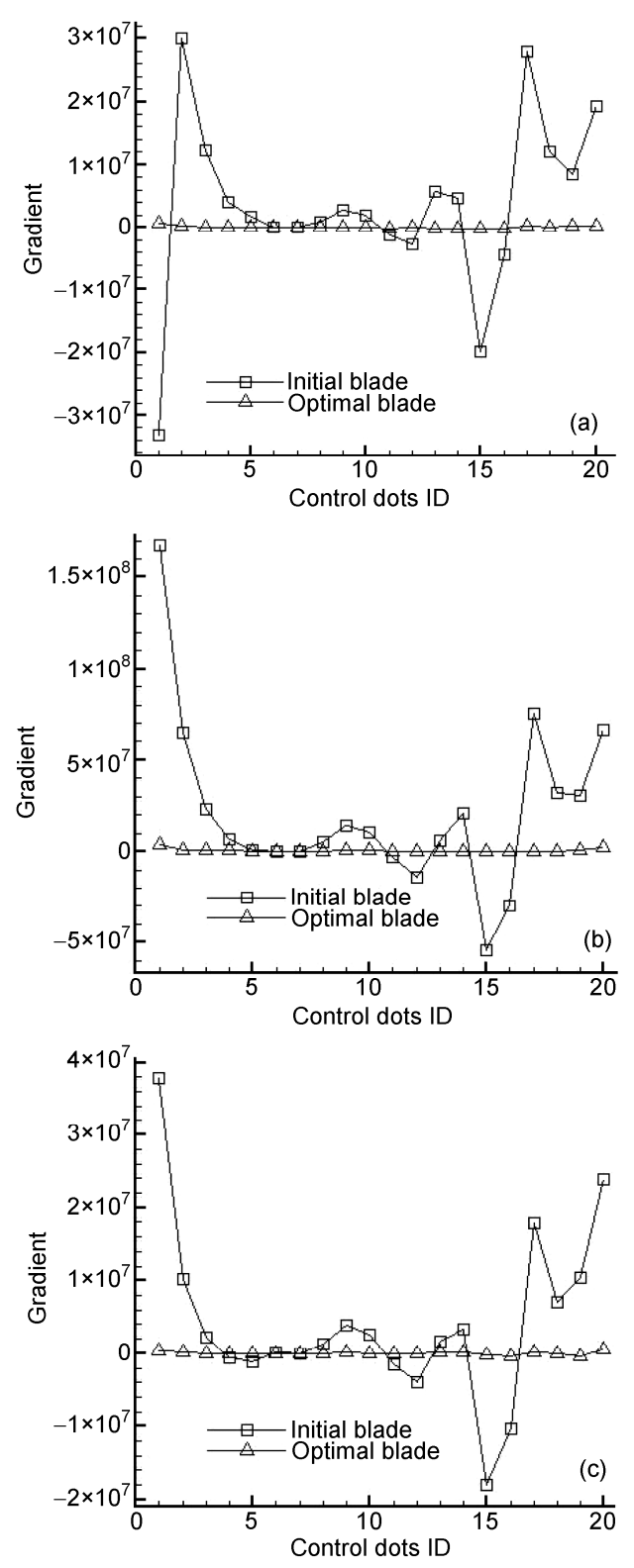

图 13 叶片初始叶型与设计叶型目标函数梯度对照图 (a) 叶片根部目标函数梯度图; (b) 叶片中径处目标函数梯度图; (c) 叶片顶部目标函数梯度图

$$
I(\alpha, p)=\frac{1}{2} \int_{\text {blade }}\left(p-p_{\mathrm{d}}\right)^{2} \mathrm{~d} s .
$$

引入本文研发的伴随系统, 最终可得如下描述的透平 叶栅黏性条件下压力反设计的伴随系统(二维流动: $i=j=k=1,2, m=4$, wall=blade; 三维流动: $i=j=$ $k=1,2,3, m=5, \quad$ wall $=$ blade + hub + shroud $).$

黏性条件下叶栅压力反设计伴随方程可描述为:

$$
\frac{\partial \psi^{\mathrm{T}}}{\partial x_{i}}\left(A_{i}-A_{v i}\right)+\frac{\partial}{\partial x_{j}}\left(\frac{\partial \boldsymbol{\psi}^{\mathrm{T}}}{\partial x_{i}} B_{i j}\right)=0,
$$

其中, $A_{i}$ 的表达形式与无黏伴随方程相同，与黏性相 关的 Jacobian 矩阵 $A_{v i}$ 以及 $B_{i j}$ 可参见文献 [29].

同时, 黏性条件下压力反设计的流动变量变分 边界积分项可表示为:

$$
\begin{aligned}
& -\int_{\text {in,out }} \psi^{\mathrm{T}} A_{i} n_{i} \delta \omega \mathrm{d} S+\int_{\text {blade }}\left(p-p_{d}\right) \delta p \mathrm{~d} S \\
& -\int_{\text {wall }}\left[\psi_{i+1}\left(n_{i} \delta p-\delta \tau_{i n}\right)-\kappa \psi_{m} \delta\left(\frac{\partial T}{\partial n}\right)+\kappa \frac{\partial \psi_{m}}{\partial n} \delta T\right] \mathrm{d} S=0 .
\end{aligned}
$$

在固壁边界上, 由于 (32)式中含有 $\delta \tau_{\text {in }}$ 项, 因此 对于给定的黏性条件下透平叶桷压力反设计目标函 数式(30)需要经过特殊处理, 才能够使得(32)式成立. 定义壁面的法向黏性应力为 $\sigma_{n}=n_{i} \tau_{i n}=n_{i} n_{j} \tau_{i j}$, 并且 由文献[30]可知 $\sigma_{n}=0$ 在固体壁面上恒成立, 从而目 标函数变分最终可改写为:

$$
\begin{aligned}
\delta I= & \int_{\text {blade }}\left(p-p_{\mathrm{d}}\right) n_{i}\left(n_{i} \delta p-\delta \tau_{\text {in }}\right) \mathrm{d} S \\
& +\frac{1}{2} \int_{\text {blade }}\left(p-p_{\mathrm{d}}\right)^{2} \delta(\mathrm{d} S) \\
& -\int_{\text {blade }}\left(p-p_{\mathrm{d}}\right) \tau_{i n} \delta n_{i} \mathrm{~d} S .
\end{aligned}
$$

经过以上特殊处理后, 黏性条件下压力反设计 伴随方程的边界条件统一表达式可描述为:

$$
\begin{aligned}
& -\int_{\text {in,out }} \psi^{\mathrm{T}} A_{i} n_{i} \delta \omega \mathrm{d} S+\int_{\text {blade }}\left(p-p_{\mathrm{d}}\right) n_{i}\left(n_{i} \delta p-\delta \tau_{\text {in }}\right) \mathrm{d} S \\
& -\int_{\text {wall }}\left[\psi_{i+1}\left(n_{i} \delta p-\delta \tau_{\text {in }}\right)-\kappa \psi_{m} \delta\left(\frac{\partial T}{\partial n}\right)+\kappa \frac{\partial \psi_{m}}{\partial n} \delta T\right] \mathrm{d} S=0 .
\end{aligned}
$$

并且目标泛函最终变分为:

$$
\begin{aligned}
\delta J= & -\int_{\text {wall }}\left[\psi_{i+1} p-\boldsymbol{\psi}^{\mathrm{T}}\left(\boldsymbol{f}_{i}-\boldsymbol{f}_{v i}\right)\right] \delta n_{i} \mathrm{~d} S \\
& -\int_{\text {blade }}\left(p-p_{\mathrm{d}}\right) \tau_{i n} \delta n_{i} \mathrm{~d} S+\frac{1}{2} \int_{\text {blade }}\left(p-p_{\mathrm{d}}\right)^{2} \delta(\mathrm{d} S) \\
& +\oint_{\partial \Omega} \boldsymbol{\psi}^{\mathrm{T}} \frac{\partial\left(\boldsymbol{f}_{i}-\boldsymbol{f}_{v i}\right)}{\partial x_{k}} n_{i} \delta x_{k} \mathrm{~d} S \\
& +\oint_{\partial \Omega} \frac{\partial \boldsymbol{\psi}^{\mathrm{T}}}{\partial x_{i}} B_{i j} \frac{\partial \boldsymbol{\omega}}{\partial x_{k}} n_{j} \delta x_{k} \mathrm{~d} S .
\end{aligned}
$$

\section{1) 二维平面叶栅压力反设计}

本算例的叶型几何及气动参数如表 3 所示. 二维 叶栅的吸力面与压力面均采用非均匀 $\mathrm{B}$ 样条来参数 化, 如图 14 所示. 图 15 给出了其计算网格的示意图. 


\section{表 3 二维叶栅的部分几何及流动参数}

\begin{tabular}{lc}
\hline \multicolumn{1}{c}{ Item } & Value \\
\hline Inlet total pressure $(\mathrm{Pa})$ & 344740.0 \\
Inlet total temperature $(\mathrm{K})$ & 709.0 \\
Outlet static pressure $(\mathrm{Pa})$ & 206431.0 \\
Pitch $(\mathrm{m})$ & 0.0582 \\
Inlet flow angle $\left(^{\circ}\right)$ & 90.0 \\
\hline
\end{tabular}

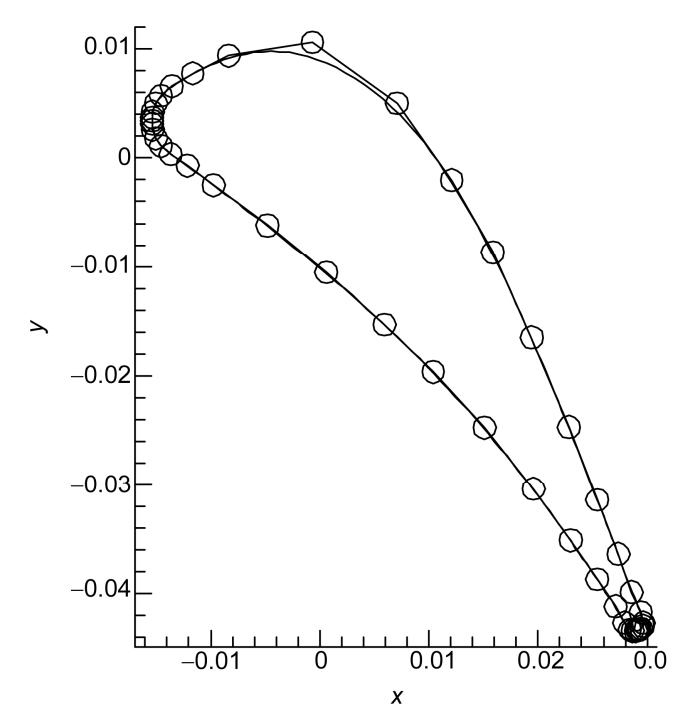

图 14 初始叶型及其 B 样条控制点

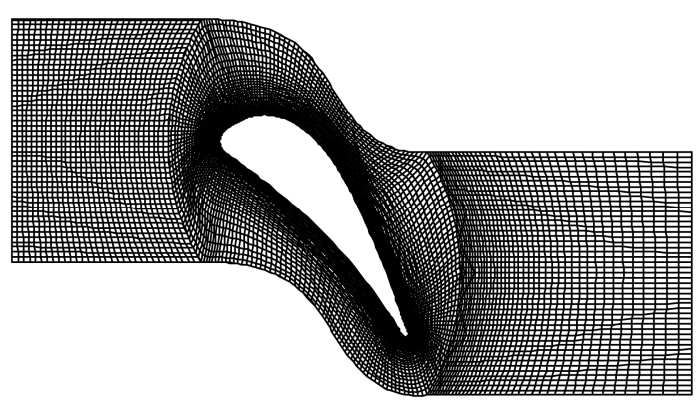

图 15 计算网格示意图

由于基于梯度的优化方法为局部寻优, 故优化 过程分三步进行, 每步限制其可调控制点区间. 优化 过程中, 假设一些几何参数包括叶宽、节距、进出口 角度、进出口楔角、前尾缘半径和出口截面积为固定 值, 计算给定的初始叶型所对应的壁面压力分布, 人 工修改压力分布并作为目标压力从而达到提高叶栅 性能的目的, 分多步适当的选取吸力面上 B 样条控 制点为可变控制点进行反设计优化.

Step 1. 控制点选为吸力面第 7 至第 18 点.
共进行了 9 次优化迭代, 选取了 24 个控制变量, 见图 16(a), 进行了 22 次流场计算便进入了局部极值 点, 图 16 中给出了此时的压力分布对照图, 最优叶 型压力分布开始向目标压力分布贴合.

Step 2. 控制点选为吸力面第 9 和 10 点.

本步优化着重点集中于吸力面喉部之前, 选取 可调控制点为吸力面 9 和 10 号点, 见图 16(b). 一共 有 4 个设计变量, 优化迭代进行了 9 步, 流场计算了 27 次便陷入了局部极值点. 图中给出了 Step 2 时的 压力分布对照图, 可以看出, 经过此步的优化, 最优 叶型压力分布与目标压力分布贴合得较好, 但在叶 片尾部扩压段压力还有所波动.

Step 3. 控制点选为吸力面第 11 至 18 点.

设计变量共有 16 个, 优化迭代 20 步收敛, 流场 计算了 30 次. 图 16(c)给出了本步可调控制点区间, 图中也给出了 Step 3 时的压力分布对照图, 通过比较 可以发现, 最终优化得到的叶型压力分布与目标压 力分布吻合得很好, 满足了设计需求.

通过优化过程可以发现, 基于控制理论二维叶 栅反设计方法的优越性在于其计算精度较高及计算 时间耗费较少. 此外, 基于梯度的优化算法是一种局 部寻优的过程, 为了避免陷入局部极值点, 有必要对 优化过程分多步进行, 每步限制可调控制点选取区 间, 从而顺利跳出某极值点. 这里需要特别指出的是 如何给定目标压力分布是一个很难的任务, 这取决 于设计人员对于叶栅气动理论及设计经验的掌握, 当设计人员所给定的目标压力分布没有实际物理意 义, 即理论上没有对应的叶型与之匹配时, 反设计优 化程序则无法收敛到与压力分布对应的目标叶型.

\section{2) 三维环形叶栅压力反设计}

以某三维叶栅为例, 进行压力反设计程序的验 证. 基于原始叶栅根部、中径处及顶部叶型, 应用非 均匀 B 样条曲面进行拟合, 并选取径向 5 个截面的部 分控制点作为设计变量, 改变其 $x y$ 方向的坐标得到 初始叶片, 如图 17 所示. 设计过程中保证前后缘及 叶片径向扭转不变, 选取 5 个截面上吸力面、压力面 分别为 5 个及 2 个可调控制点的 $x y$ 坐标, 共计 70 个 设计变量. 目标压力分布通过对原有叶栅流场的数 值模拟得到，其设计条件如表 4 所示.

设计过程中 N-S 方程求解器使用 FINE 7.21, 采 用中心差分格式及 B-L 湍流模型求解雷诺时均 N-S 方程, 并采用多重网格及当地时间步长加速收玫. 计 


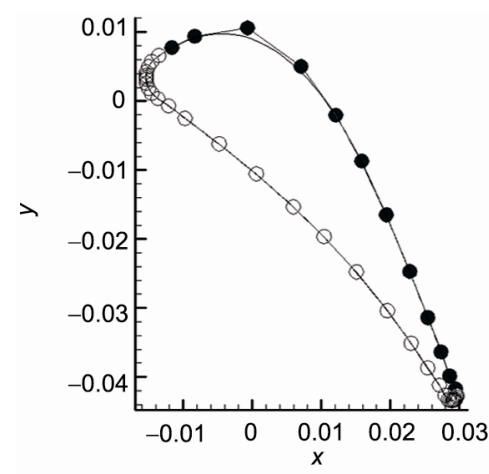

(a1) Step 1

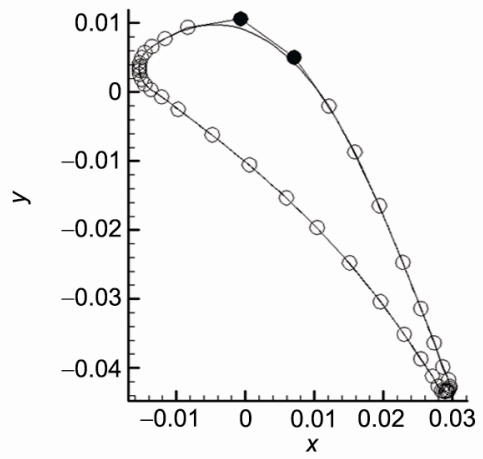

(b1) Step 2

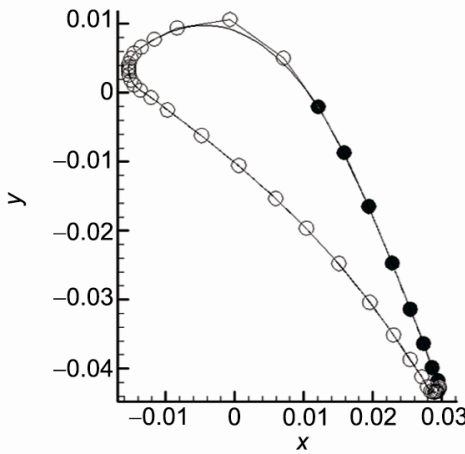

(c1) Step 3

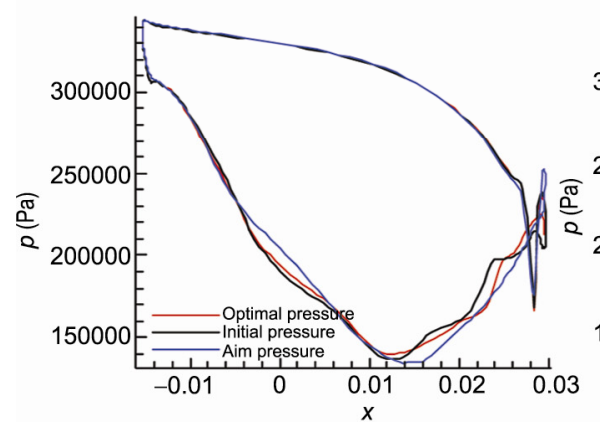

(a2) Step 1

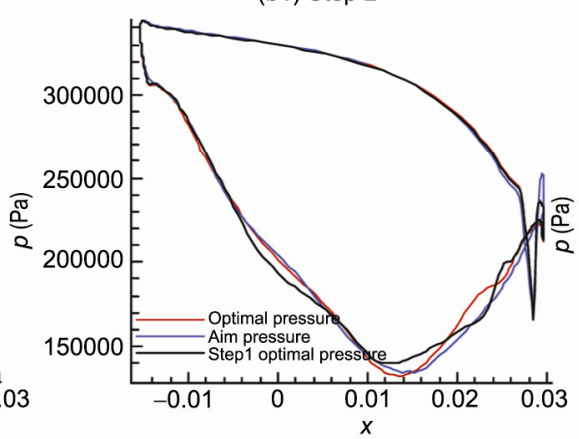

(b2) Step 2

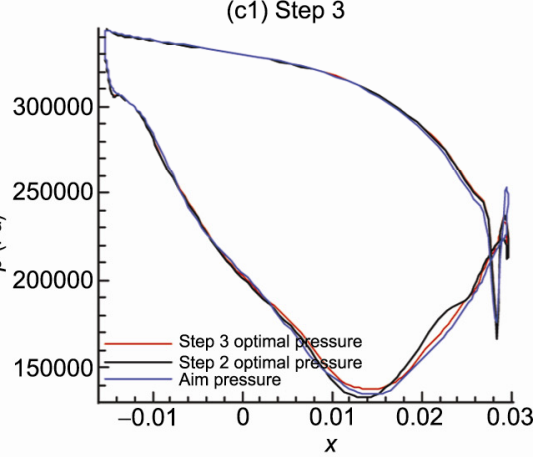

(c2) Step 3

图 16 不同 Step 时的叶型控制点选取示意图与压力分布对照图

(a) Step1; (b) Step 2; (c) Step 3

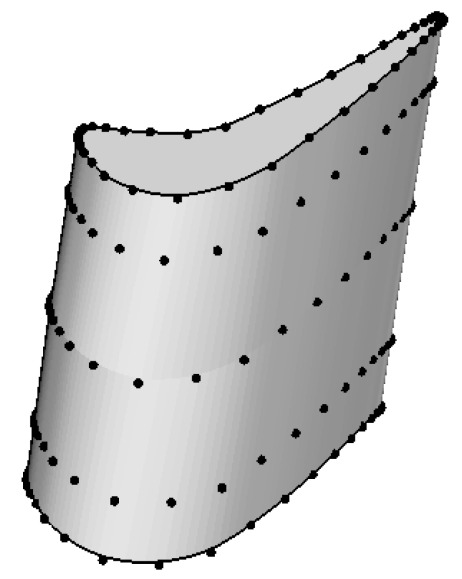

图 17 初始叶型及控制点

表 4 三维叶栅部分几何及气动参数

\begin{tabular}{lc}
\hline \multicolumn{1}{c}{ Item } & Value \\
\hline Inlet total pressure $(\mathrm{Pa})$ & 224929.0 \\
Inlet total temperature $(\mathrm{K})$ & 627.65 \\
Number of blades & 76 \\
Middle diameter $(\mathrm{mm})$ & 344.70 \\
Blade height $(\mathrm{mm})$ & 42.65 \\
Outlet static pressure $(\mathrm{Pa})$ & 206431.0 \\
Inlet flow angle $\left(^{\circ}\right)$ & 90.0 \\
\hline
\end{tabular}

算区域采用 AUTOGRID 软件生成 H-I 网格, 网格数 为 $77 \times 21 \times 25$, 如图 18 所示. 该算例经过 37 步迭代后 收敛至局部极值点, 共进行了 44 次流场计算.

图 19 和 20 分别给出了根部、中径处及顶部截面 的初始叶型、设计叶型及参考叶型的比较, 以及近根 部、中径处及近顶部压力分布的比较. 图中可看出反 设计的叶片与参考叶片吻合很好, 压力分布与目标基 本吻合. 图 21 给出了设计迭代的无量刚目标函数下降 过程, 经 37 步迭代后目标函数值下降了 2 个数量级, 并不再有明显的下降，可看出已收敛至局部最优值.

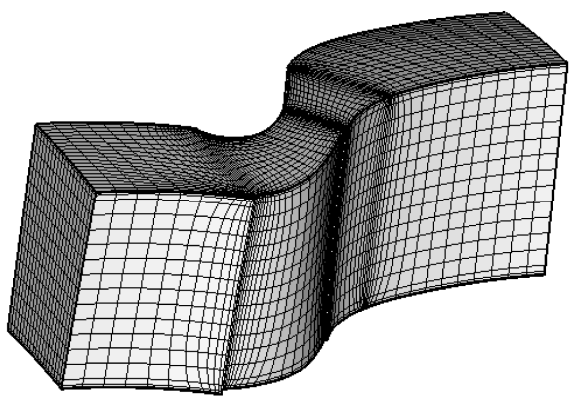

图 18 计算区域 H-I 型网格 


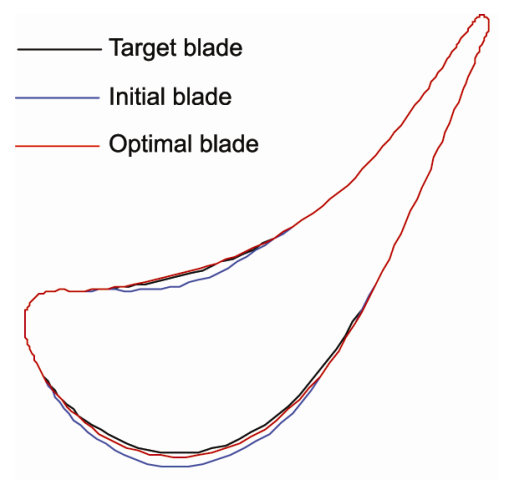

(a) Hub

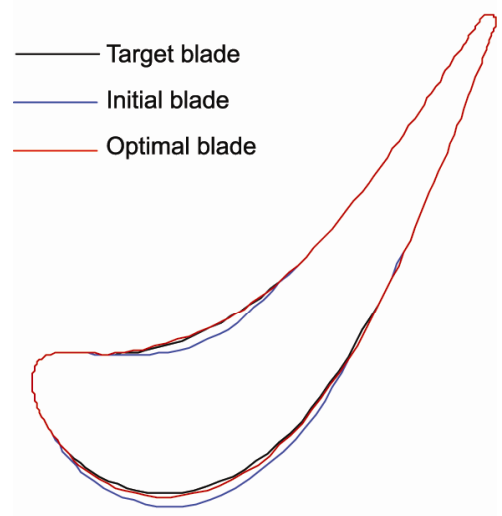

(b) Mid-span

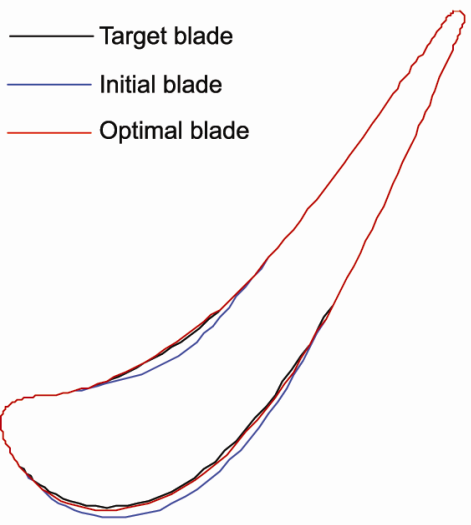

(c) Shroud

图 19 三维叶栅典型截面上的叶型比较

(a) 叶根处; (b) 中叶展处; (c) 叶顶处

\section{3 透平叶栅等熵马赫数反设计优化}

给定叶栅壁面的目标等熵马赫数分布 $M a_{i s}^{d}$, 则轴 流式透平叶栅等熵马赫数反设计目标函数可定义为

$$
I=\frac{1}{2} \int_{\text {blade }}\left(M a_{i s}-M a_{i s}^{d}\right)^{2} \mathrm{~d} S .
$$

如前所述, 对于黏性流体, 壁面处的法向黏性应
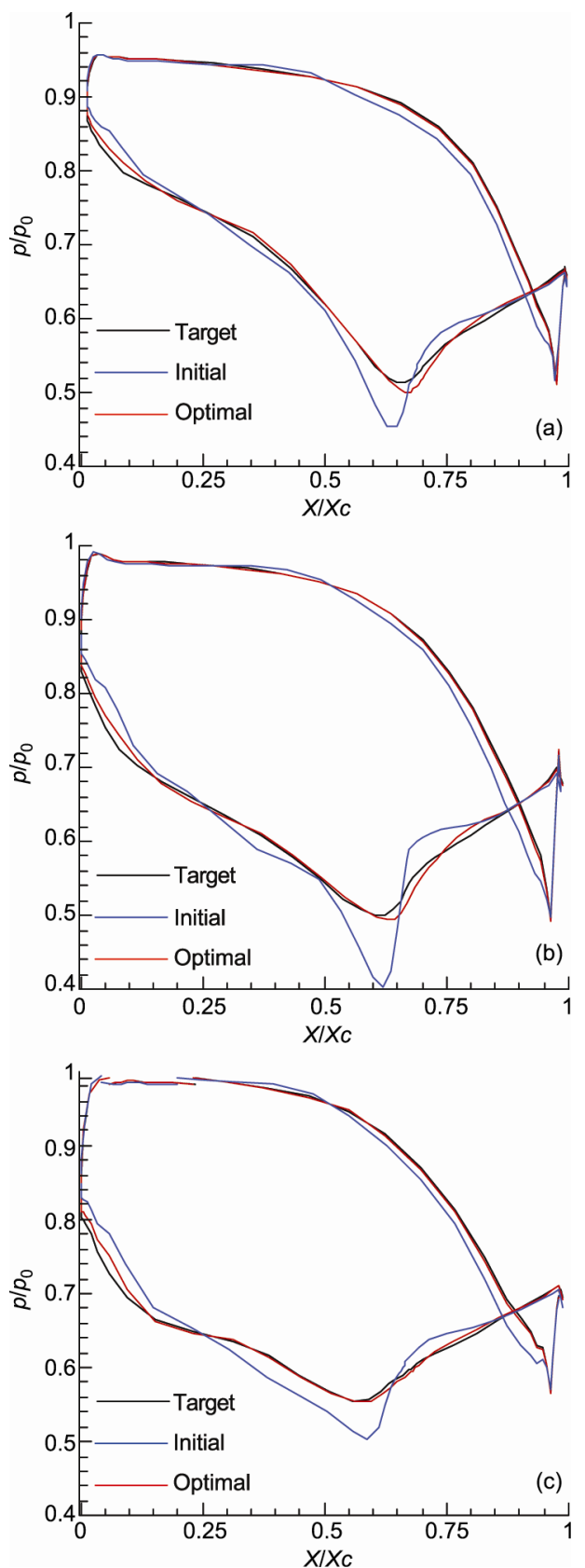

图 20 三维叶栅典型截面上的压力分布比较

(a) 在根部截面; (b) 在中径处截面; (c) 在顶部截面

力 $\sigma_{n}=0$ 恒成立, 因此目标函数变分式可表述为:

$$
\begin{aligned}
\delta I= & \int_{\text {blade }}\left[\Pi n_{i}\left(n_{i} \delta p-\delta \tau_{i n}\right)-\Pi \tau_{i n} \delta n_{i}\right] \mathrm{d} S \\
& +\frac{1}{2} \int_{\text {blade }}\left(M a_{i s}-M a_{i s}^{d}\right)^{2} \delta(\mathrm{d} S),
\end{aligned}
$$

其中, $\Pi=\frac{1}{\gamma p_{0} M a_{i s}}\left(\frac{p}{p_{0}}\right)^{\frac{1-2 \gamma}{\gamma}}\left(M a_{i s}-M a_{i s}^{d}\right), p_{0}$ 为入口 


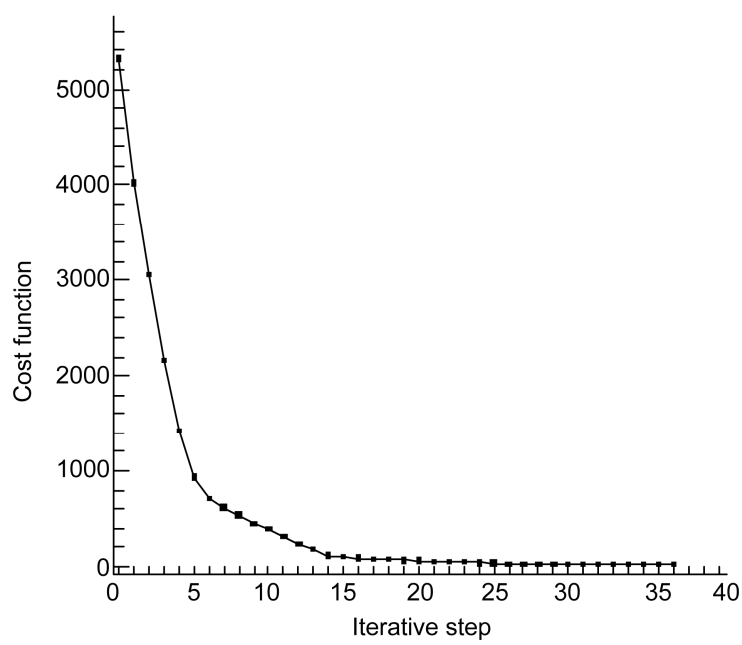

图 21 迭代收敛史

总压, $p$ 为叶栅壁面压力.

结合本文提出的一般性气动反设计问题的伴随 系统，最终目标函数对设计变量的梯度可描述如下：

$$
\begin{aligned}
\frac{\mathrm{d} J}{\mathrm{~d} \alpha}= & \int_{\text {wall }} \frac{\partial \boldsymbol{\psi}^{\mathrm{T}}}{\partial x_{i}} B_{i j} \frac{\partial \boldsymbol{\omega}}{\partial x_{k}} n_{j} \frac{\delta x_{k}}{\delta \alpha} \mathrm{d} S-\int_{\text {blade }} \Pi \tau_{i n} \frac{\delta n_{i}}{\delta \alpha} \mathrm{d} S \\
& +\int_{\text {wall }} \boldsymbol{\psi}^{\mathrm{T}} \frac{\partial\left(\boldsymbol{f}_{i}-\boldsymbol{f}_{v i}\right)}{\partial x_{k}} n_{i} \frac{\delta x_{k}}{\delta \alpha} \mathrm{d} S+\frac{1}{2} \int_{\text {blade }}\left(M a_{i s}\right. \\
& \left.-M a_{i s}^{d}\right)^{2} \frac{\delta(\mathrm{d} S)}{\delta \alpha}-\int_{\text {wall }}\left[\psi_{i+1} p-\boldsymbol{\psi}^{\mathrm{T}}\left(\boldsymbol{f}_{i}-\boldsymbol{f}_{v i}\right)\right] \frac{\delta n_{i}}{\delta \alpha} \mathrm{d} S .
\end{aligned}
$$

为验证轴流式透平叶栅等熵马赫数反设计程序, 以某二维平面叶栅为例, 应用非均匀 $\mathrm{B}$ 样条进行叶 型的参数化, 选取吸力面上的 8 个样条控制点为设计 变量, 见图 22 所示. 表 5 给出了叶栅的部分参数.

在设计优化过程中, 假设一些几何参数包括叶 宽、节距、进出口角度、进出口楔角、前尾缘半径和 出口截面积为固定, 计算给定的初始叶型所对应的 壁面等摘马赫数分布, 人工修改等熵马赫数分布并 作为目标分布规律从而达到提高叶栅性能的目的, 适当的选取吸力面上 $\mathrm{B}$ 样条控制点为可变控制点来 进行反设计优化. 反设计过程中保证前后缘及叶片 径向扭转不变. 其中, 为了增加几何约束, 截面上的 叶背型线以喉部切分为两段, 反设计过程中保持喉部 控制点不动. 选取吸力面上的 8 个可调控制点(其中喉 部点之前 5 个, 喉部点之后 3 个)沿法线方向移动距离 作为设计变量, 如图 22 所示, 共计 8 个设计变量.

整个计算过程进行了 76 次优化循环便得到了最
优叶型, 计算条件为个人 $\mathrm{PC}(3.0 \mathrm{GHz} \mathrm{CPU}, 2 \mathrm{~GB}$ Memory), 耗时约 $24 \mathrm{~h}$. 图 23 给出了最优叶型与初始 叶型对照图, 可以看出, 相对于原始叶型, 最优叶型 厚度分布更为均匀、型线更为光顺. 图 24 给出了初

\section{表 5 二维叶栅部分几何及流动参数}

\begin{tabular}{lc}
\hline \multicolumn{1}{c}{ Item } & Value \\
\hline Inlet total pressure $(\mathrm{Pa})$ & 2489000.0 \\
Inlet total temperature $(\mathrm{K})$ & 1600.0 \\
Outlet static pressure $(\mathrm{Pa})$ & 114000.0 \\
Pitch $(\mathrm{m})$ & 0.282 \\
Inlet flow angle $\left(^{\circ}\right)$ & 90.0 \\
\hline
\end{tabular}

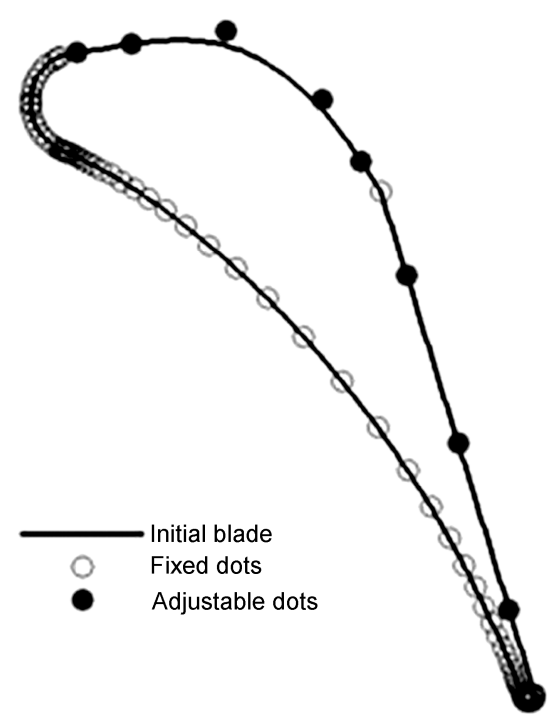

图 22 初始叶型及其控制点

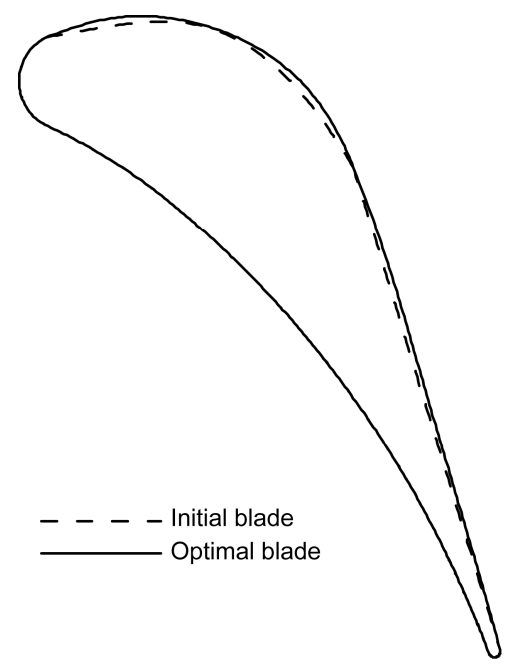

图 23 初始叶型与最优叶型对比图 


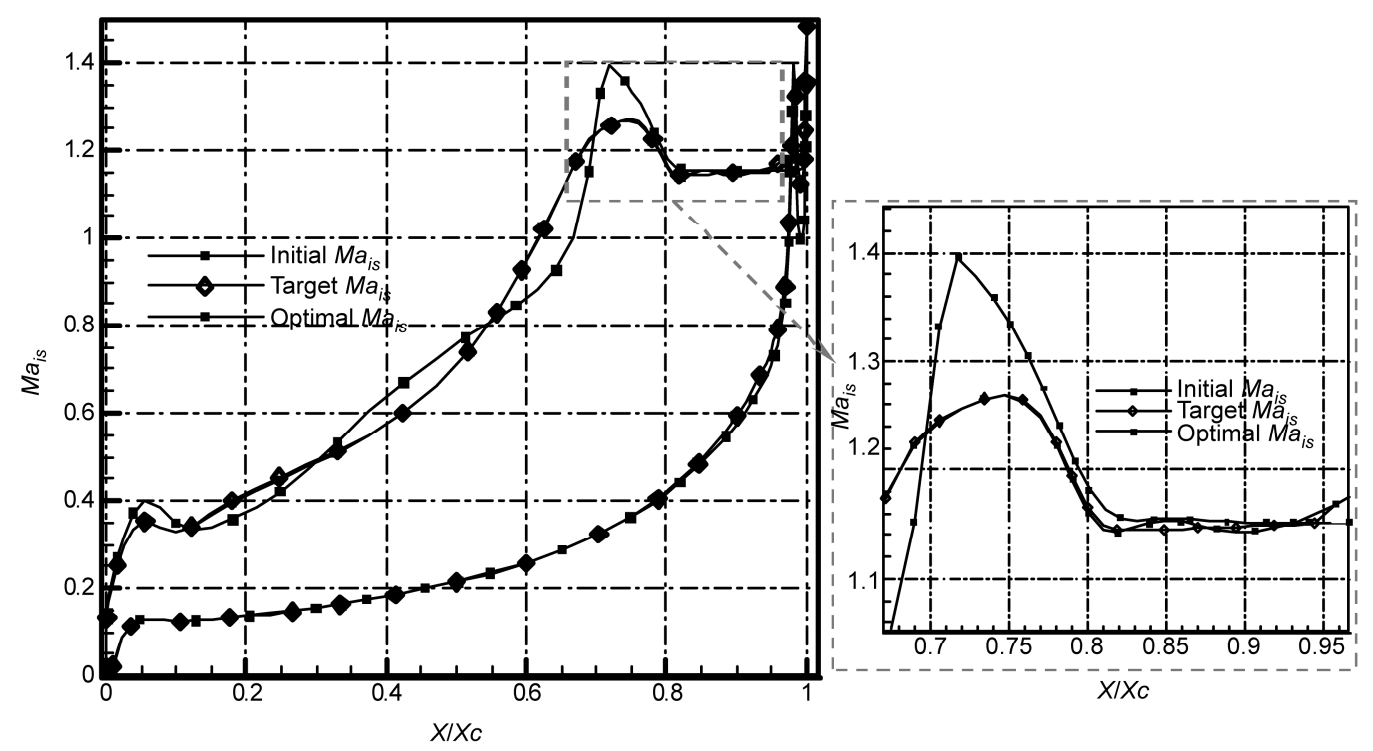

图 24 等摘马赫数分布对照图

始叶型和最优叶型与目标等熵马赫数分布的对比图, 可以看出吸力面的等摘马赫数突跳被抹平, 最优叶 片的壁面等熵马赫数分布与目标值基本吻合, 尤其 是喉部附近, 由于初始叶型曲率不连续所引起的压 力间断得到了明显改善.

\section{5 结论}

本文将控制理论应用于轴流式透平叶柱的气动 反设计优化研究中, 建立了叶栅气动反设计最优控 制模型, 开发了基于控制理论的轴流式透平叶栅气 动反设计优化系统. 在透平叶栅伴随系统的推导中 采用了网格节点位置坐标直接变分, 提出了具有普 适性意义的基于控制理论的一般性优化问题伴随系 统, 最后推导得到的目标泛函变分表达式中只包含 有网格节点位置坐标变分的边界积分项, 避免了内 部网格的重复生成和内部节点位置坐标差值计算, 进一步提高了伴随方法在梯度计算上的快速性. 伴 随系统的数值求解采用 $\mathrm{ROE}$ 格式近似黎曼通量和显 式五步龙格-库塔时间推进法, 并使用多重网格技术 和当地时间步长进行加速收敛.

同时, 本文进行了 5 个轴流式透平叶栅气动反设 计的数值算例研究, 分别包括无黏条件下二维平面 叶㮽压力反设计、无黏条件下三维环形叶㮽压力反设
计、黏性条件下二维平面叶栅压力反设计、黏性条件 下三维环形叶栅压力反设计以及黏性条件下二维平 面叶栅等摘马赫数反设计, 详细讨论了各个气动反 设计系统的伴随系统描述, 给出了伴随方程的具体表 达形式及其边界条件表达式, 对于黏性伴随系统讨论 了目标函数的限制, 通过引入壁面法向黏性应力顺利 解决了目标函数中需要的剪切应力变分. 算例研究表 明本文所研发的基于控制理论的轴流式透平叶栅气动 反设计方法与系统能够有效应用于叶栅二维、三维、 无黏及黏性条件下的气动优化反设计技术中, 为进一 步提高透平叶栅的气动性能提供了一个快速有效的反 设计优化方法与软件平台, 从而可以进一步完善透平 叶栅设计体系, 加速设计周期, 提高设计质量.

本文所提出的轴流式透平叶栅气动反设计优化 方法的特点和优点如下.

1) 与传统伴随系统建立方法相比, 本文研究建 立的连续伴随系统更为简洁、直观，大大简化了伴随 方程边界条件的推导, 并且目标泛函的最终变分中 不含网格节点坐标变分的体积分项, 避免了网格内 部节点的重复生成, 进一步提高了伴随方法在梯度 计算上的速度.

2) 结合叶型参数化方法、网格自动生成及其扰 动技术、偏微分方程数值求解技术、最优化算法, 应 用连续伴随方法开发的轴流式叶栅气动优化问题的 
伴随系统, 是基于一般性优化问题的目标函数假设, 具有很高的通用性, 能够方便有效的应用于不同的 叶栅气动优化问题.
3) 从不同的角度验证了本文所研发的伴随系统 的稳定性、收玫性和精确性, 证明该系统能够有效的 应用于轴流式透平叶栅的气动反设计优化问题中.

\section{参考文献}

1 Song L M, Feng Z P, Li J. Shape optimization of turbine stage using adaptive range differential evolution and three-dimensional NavierStokes solver. ASME Paper, GT2005-68280, 2005

2 Pierret S, Van den Braembussche R A. Turbomachinery blade design using a Navier-Stock solver and artificial neural network. ASME J Turbomachinery, 1999, 121: 326-332

3 Ahn C S, Kim K Y. Aerodynamic design optimization of a compressor rotor with Navier-Stocks analysis. Proc IMechE, Part A: J Power Energy, 2003, 217(2): 179-183

4 Pironneau O. On optimum shapes in Stokes flow. J Fluid Mech, 1973, 59(2): 117-128

5 Jameson A. Aerodynamic design via control theory. J Sci Comput, 1988, 3: 233-260

6 Jameson A, Pierce N A, Martinelli L. Optimum aerodynamic design using the Navier-Stokes equations. 35th AIAA Aerospace Sciences Meeting \& Exhibit, Reno, 1997

7 Reuther J, Jameson A, Farmer J, et al. Aerodynamic shape optimization of complex aircraft configurations via an adjoint formulation. AIAA Paper 96-0094, 1996

8 Elliott J, Peraire J. 3D aerodynamic optimization on unstructured meshes with viscous effects. AIAA Paper 97-1849, 1997

9 Qiao Z D, Qin X L, Yang X D. Wing design by solving adjoint equations. AIAA Paper, AIAA-2002-0263, 2002

10 唐智礼. 应用控制理论的气动优化设计方法研究. 博士学位论文. 南京: 南京航空航天大学, 2000

11 Yang S, Wu H, Liu F. Aerodynamic design of cascades by using an adjoint equation method. AIAA Paper, AIAA-2003-1068, 2003

12 Wu H, Yang S, Liu F. Comparison of three geometric representation of airfoils for aerodynamic optimization. AIAA Paper, AIAA-20034095, 2003

13 Wu H, Liu F, Tsai H. Aerodynamic design of turbine blades using an adjoint equation method. AIAA Paper, AIAA-2005-1006, 2005

14 Papadimitriou D I, Giannakoglou K C. Compressor blade optimization using a continuous adjoint formulation. ASME Paper, GT200690466, 2006

15 Papadimitriou D I, Giannakoglou K C. A continuous adjoint method with objective function derivatives based on boundary integrals for inviscid and viscous flows. Computers and Fluids, 2007, 36: 325-341

16 Papadimitriou D I, Giannakoglou K C. Aerodynamic shape optimization using first and second order adjoint and direct approaches. Arch Comput Methods Eng, 2008, 15: 447-488

17 Wang D X, He L. Adjoint aerodynamic design optimization for blades in multi-stage turbomachines: Part I - methodology and verification. ASME Paper, GT2008-50208, 2008

18 Wang D X, He L. Concurrent aerodynamic-aeromechanic design optimization for turbomachinery blades using adjoint method. ASME Paper, GT2009-59240, 2009

19 Li Y C, Yang D L, Feng Z P. Inverse problem in aerodynamic shape design of turbomachinery blades. ASME Paper, GT2006-91135, 2006

20 Li Y C, Feng Z P. Aerodynamic design of turbine blades by using adjoint-based method and N-S equation. ASME Paper, GT2007-27734, 2007

21 李颖晨, 杨佃亮, 高志明, 等. 透平叶栅三维形状反问题研究. 工程热物理学报, 2007, 28(1): 33-36

22 李颖晨, 丰镇平. 透平叶栅三维黏性气动反问题的控制理论方法. 工程热物理学报, 2007, 28(4): 580-582

23 Li Y C, Feng Z P. Three-dimensional aerodynamic design of turbine blades using the adjoint method. ASME Paper, GT2008-51225, 2008

24 李砂晨, 丰镇平. 应用控制理论的叶栅气动反设计. 工程热物理学报, 2008, 29(5): 755-758

25 厉海涛, 宋立明, 李颖晨, 等. 基于控制理论和 N-S 方程的二维叶栅气动优化算法. 工程热物理学报, 2009, 30(12): 2021-2024

26 厉海涛, 宋立明, 丰镇平. 复杂约束环境下基于控制理论的透平叶栅气动优化. 工程热物理学报, 2010, 31(8): 1294-1298

27 Li H T, Song L M, Li Y C, et al. 2D viscous aerodynamic shape design optimization for turbine blades based on adjoint method. ASME Paper, GT2009-59999, 2009; also ASME J Turbomachinery, 2011, 133(3): 031014

28 Blazek, J. Computational Fluid Dynamics: Principles and Applications. Elsevier, 1st Edition, 2001

29 李颖晨. 基于控制理论的轴流式叶栅气动反设计研究. 博士学位论文. 西安: 西安交通大学, 2008

30 Li H T, Song L M, Zhang P F, et al. Inverse problem for isentropic Mach-number on blade wall in aerodynamic shape design of turbomachinery cascades by using adjoing method. ASME Pager, GT2011-45808, 2011 